\title{
IRF4 instructs effector Treg differentiation and immune suppression in human cancer
}

\author{
Giorgia Alvisi, ${ }^{1}$ Jolanda Brummelman, ${ }^{1}$ Simone Puccio, ${ }^{1}$ Emilia M.C. Mazza, ${ }^{1}$ Elisa Paoluzzi Tomada, ${ }^{1}$ Agnese Losurdo, ${ }^{2}$ \\ Veronica Zanon, ${ }^{1}$ Clelia Peano, ${ }^{3,4}$ Federico S. Colombo, ${ }^{5}$ Alice Scarpa, ${ }^{1}$ Marco Alloisio, ${ }^{6,7}$ Ajithkumar Vasanthakumar, ${ }^{8}$ \\ Rahul Roychoudhuri, ${ }^{9}$ Marinos Kallikourdis, ${ }^{10}$ Massimiliano Pagani, ${ }^{11}$ Egesta Lopci, ${ }^{12}$ Pierluigi Novellis, ${ }^{6}$ \\ Jonas Blume, ${ }^{8}$ Axel Kallies, ${ }^{8}$ Giulia Veronesi, ${ }^{6}$ and Enrico Lugli ${ }^{1}, 5$
}

'Laboratory of Translational Immunology, Humanitas Clinical and Research Center, Rozzano, Milan, Italy. ${ }^{2 H u m a n i t a s ~ C l i n i c a l ~ a n d ~ R e s e a r c h ~ C e n t e r ~-~ I R C C S, ~ H u m a n i t a s ~ C a n c e r ~ C e n t e r, ~ R o z z a n o, ~ M i l a n, ~}$ Italy. ${ }^{3}$ Division of Cenetic and Biomedical Research, UOS Milan, National Research Council, Rozzano, Milan, Italy. ${ }^{4}$ Cenomic Unit and ${ }^{5}$ Humanitas Flow Cytometry Core, Humanitas Clinical and Research Center, Rozzano, Milan, Italy. ${ }^{6}$ Division of Thoracic Surgery, Humanitas Clinical and Research Hospital, Rozzano, Milan, Italy. ${ }^{7}$ Biomedical Science Department, Humanitas University, Rozzano, Milan, Italy. ${ }^{8}$ Department of Microbiology and Immunology, The Peter Doherty Institute for Infection and Immunity, University of Melbourne, Melbourne, Victoria, Australia. ${ }^{9}$ Laboratory of Lymphocyte Signalling and Development, Babraham Institute, Cambridge, United Kingdom. ${ }^{10}$ Adaptive Immunity Laboratory, Humanitas Clinical and Research Center, Rozzano, Milan. "Istituto Nazionale Genetica Molecolare "Romeo ed Enrica Invernizzi," Milan, Italy. ${ }^{2}$ Nuclear Medicine Department, Humanitas Clinical and Research Hospital, Rozzano, Milan, Italy.

\begin{abstract}
The molecular mechanisms responsible for the high immunosuppressive capacity of CD4+ Tregs in tumors are not well known. High-dimensional single-cell profiling of T cells from chemotherapy-naive individuals with non-small-cell lung cancer identified the transcription factor IRF4 as specifically expressed by a subset of intratumoral CD4+ effector Tregs with superior suppressive activity. In contrast to the IRF4- counterparts, IRF4+ ${ }^{+}$Tregs expressed a vast array of suppressive molecules, and their presence correlated with multiple exhausted subpopulations of T cells. Integration of transcriptomic and epigenomic data revealed that IRF4, either alone or in combination with its partner BATF, directly controlled a molecular program responsible for immunosuppression in tumors. Accordingly, deletion of Irf4 exclusively in Tregs resulted in delayed tumor growth in mice while the abundance of IRF4+ ${ }^{+}$Tregs correlated with poor prognosis in patients with multiple human cancers. Thus, a common mechanism underlies immunosuppression in the tumor microenvironment irrespective of the tumor type.
\end{abstract}

\section{Introduction}

Despite recent clinical breakthroughs in adoptive T cell transfer approaches and checkpoint blockade in treating hematopoietic and solid tumors, suppression of the antitumor immune response in the tumor microenvironment (TME) is a major obstacle to tumor regression (1). Although antitumor immune cells, such as T and NK cells, normally infiltrate tumors, and their abundance correlates with better prognosis in certain cancers, the presence of inhibitory populations, such as regulatory $\mathrm{CD}^{+} \mathrm{T}$ cells (Tregs), counteract tumor rejection (2). The main role of Tregs is to maintain immune homeostasis in physiology by inhibiting effector $\mathrm{T}$ cells via different modes of action, including expression of cell-surface inhibitors, such as cytotoxic T lymphocyte-associated protein 4 (CTLA-4) (3); production of inhibitory cytokines, such as IL-10 (4), TGF- $\beta$ (5), or IL-35 (6); depletion of IL-2 by overexpression of the high-affinity IL-2

Authorship note: СA, J. Brummelman, and SP share first authorship. AK and GV contributed equally to this work.

Conflict of interest: The Laboratory of Translational Immunology receives reagents in-kind from BD Biosciences Italy as part of a collaborative research agreement. Copyright: () 2020, American Society for Clinical Investigation.

Submitted: May 28, 2019; Accepted: February 26, 2020; Published: May 11, 2020.

Reference information: / Clin Invest. 2020;130(6):3137-3150.

https://doi.org/10.1172/JCl130426. receptor $\alpha$ chain (IL-2R $\alpha$, also known as CD25); purine-mediated suppression by CD39-dependent conversion of ATP (7); or direct cytotoxicity (8), thus preventing autoimmunity and immunopathology (reviewed in Vignali et al., ref. 9). Conversely, Tregs are aberrantly enriched in tumors and dampen antitumor immune responses (10). This detrimental effect on antitumor immunity is demonstrated by a plethora of preclinical data in which systemic Treg depletion or inhibition of their function promotes tumor regression (11-14). Unfortunately, depletion or inhibition of Tregs often results in severe autoimmunity, allergy, and immunopathology (11-14); therefore, new strategies are required to safely deplete Tregs specifically in the TME. The prerequisite to do so is the identification of a specific molecular program or molecules that are exclusively present in tumorassociated Tregs, which would therein allow us to spare circulating Tregs and maintain peripheral homeostasis.

Transcriptional profiling recently revealed that $\mathrm{CD} 4^{+}$Tregs isolated from colon, lung, and breast tumors are transcriptionally different from those isolated from the adjacent tissues and the blood $(15,16)$. In particular their transcriptional profile overlaps with that of effector Tregs described in mice, which show enhanced suppressive activity. This includes the increased expression of molecules, such as CTLA-4, 4-1BB, CCR8, ICOS, and others (17). Noteworthily, increased expression of CCR $8(15,16), L A Y N$, or MAGEH1 (15) by the intratu- 
A

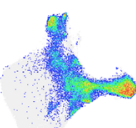

Blood

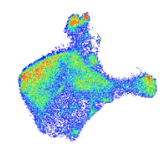

Lung Tissue

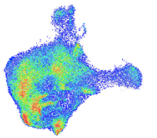

Tumor
B

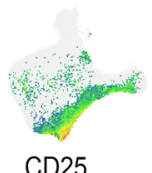

CD25

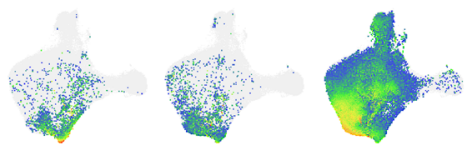

FOXP3

IRF4

PD1

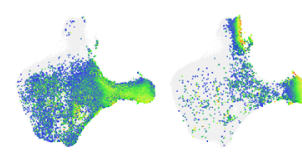

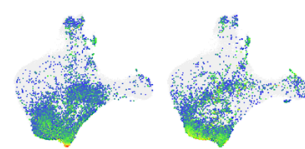

TIGIT

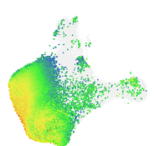

CD69
MFI of positive cells Max [Min
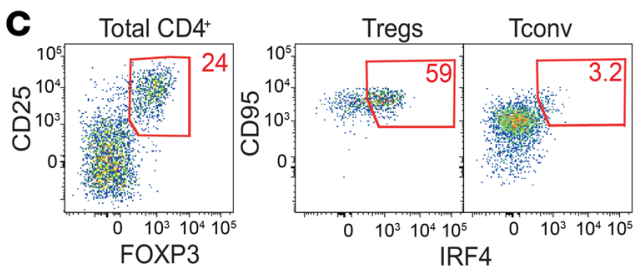

F

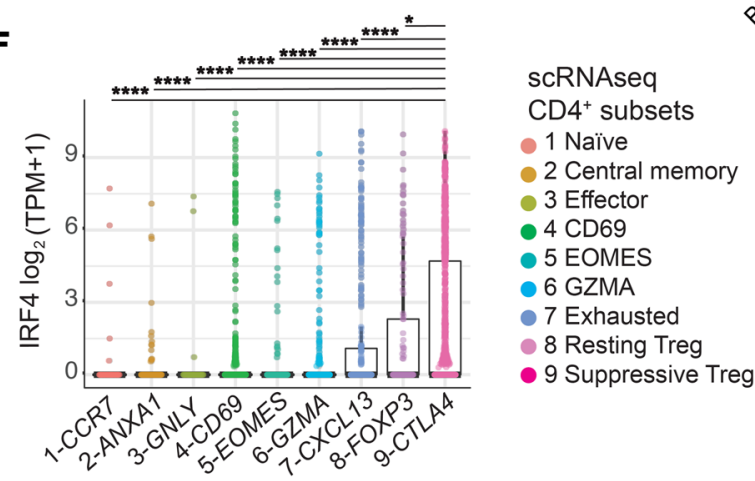

G

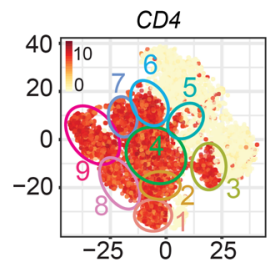

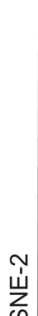

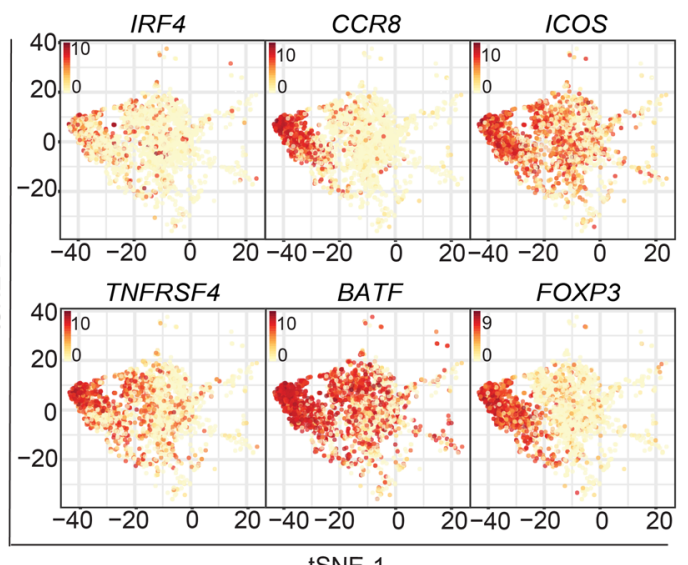

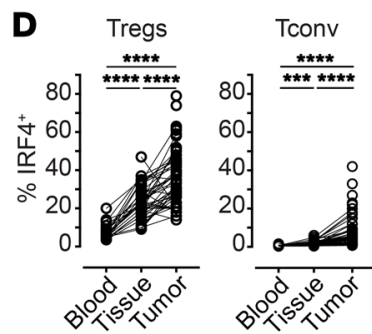

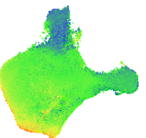

CD98

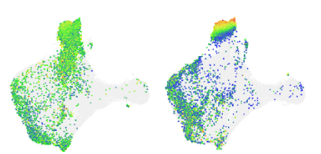

T-bet

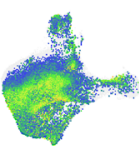

CXCR3

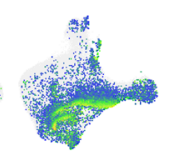

CXCR5
E
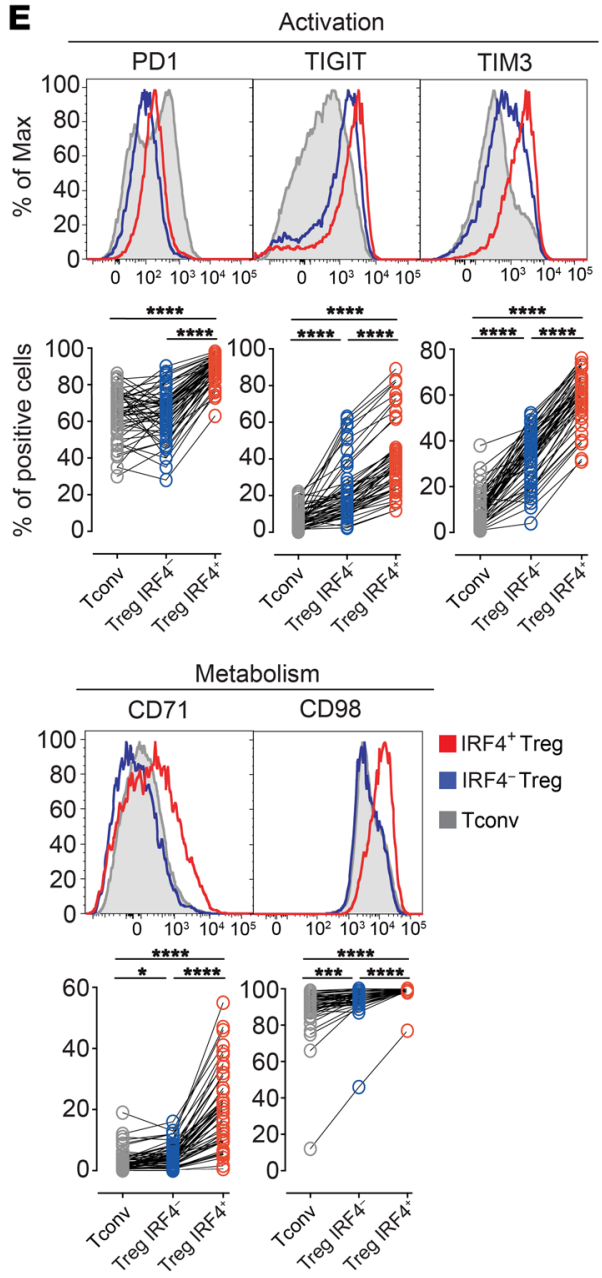
Figure 1. IRF4 identifies effector Tregs enriched in human tumors. (A) UMAP analysis of concatenated CD4+ $\mathrm{T}$ cells (1,500 cells/sample) from tumor $(n=53)$, adjacent lung tissue $(n=45)$, and blood $(n=22)$ samples from patients with NSCLC. (B) UMAP of relative marker expression by concatenated $\mathrm{CD}^{+} \mathrm{T}$ cells from the same samples in A. (C) Manual gating analysis of $\mathrm{CD}^{+}{ }^{+} \mathrm{CD} 25^{+} \mathrm{FOXP3}^{+}$Tregs expressing IRF4 ${ }^{+}$by flow cytometry. Numbers indicate the percentage of positive cells. (D) Summary plot representing the IRF4 expression in CD4+ Tregs and conventional T (Tconv cells; defined as $\left.\mathrm{CD}_{25} 5^{\circ} \mathrm{FoxP3} 3^{-}\right)$cells from the same patients as in $\mathbf{A}\left({ }^{* * *} P<\right.$ $0.0005,{ }^{* * *} P<0.0001$, Kruskal-Wallis test). (E) Representative distribution by flow cytometry (top) and summary of the percentage of expression of selected markers (bottom) in tumor-infiltrating IRF4+ and IRF4- Tregs and Tconv cells $\left({ }^{*} P<0.01\right.$, ${ }^{* * *} P<0.0005$, ${ }^{* * *} P<0.0001$, nonparametric Friedman test). (F) Box plot showing the $\log _{2}(T P M+1)$ expression of IRF4 transcript across 9 CD4 ${ }^{+} T$ cells clusters as identified by single-cell RNA-seq (25). Each dot represents a single cell $\left({ }^{*} P \leq 0.01,{ }^{* * *} P \leq 0.0001\right.$, Wilcoxon test). (C) t-SNE plots illustrating the expression of selected genes in single $\mathrm{CD}^{+} \mathrm{T}$ cells from lung tumor lesions. Cell clusters, depicted on the left, were identified as in $\mathbf{F}$.

moral Tregs correlated with poor prognosis, suggesting a pivotal role of these cells in inhibiting antitumor immune responses and/or favoring tumor growth.

The molecular mechanisms leading to increased Treg activity in tumors remain ill-defined. Studies performed over the last decade have elucidated in part the transcriptional network at the basis of murine effector Treg differentiation in lymphoid and nonlymphoid tissues. Once activated, Tregs undergo a program of effector differentiation that mirrors Th cell differentiation by expressing transcription factors (TFs) that regulate Th polarization, such as T-bet, GATA-3, or Bcl-6 (17). Additional TFs might be involved in regulating effector Tregs at specific tissue sites, such as PPAR $\gamma$, which controls the unique transcriptional and metabolic signature of those cells residing in the visceral adipose tissue (18). Interferon regulatory factor 4 (IRF4), initially identified as the TF responsible for the generation of those Tregs specifically controlling Th2 responses (19), plays a central role in generating effector Tregs in peripheral organs (20). IRF4 is absent in quiescent $\mathrm{T}$ cells but is induced by $\mathrm{T}$ cell receptor (TCR) signaling. Mice with specific deletion of Irf4 in Tregs develop multiorgan autoimmunity due to exacerbated Th1, Th17, and Tfh responses and plasma cell infiltration (19). Notably, IRF4-deficient Tregs largely maintain a CD62 $\mathrm{L}^{\text {hi }}$ naive-like phenotype and express reduced amounts of effector and suppressive molecules, such as ICOS, CTLA-4, and IL-10 (20). IRF4 cooperates with other TFs in DNA binding and regulating transcription in CD4 $4^{+}$ T cells, namely the AP- 1 family members BATF and JUN $(21,22)$. Notably, IRF 4 was reported to be overexpressed by non-smallcell lung cancer-infiltrating (NSCLC-infiltrating) Tregs (23), but its role in determining the Treg transcriptional program, functionality, and regulation of antitumor immunity remains completely unexplored.

Here, we reported the existence of 2 subsets of tumor-infiltrating Tregs with differential expression of IRF4, as revealed by high-dimensional single-cell analysis of lung, liver, and melanoma-infiltrating T cells. IRF4 defined activated effector Tregs with enhanced suppressive activity, the abundance of which correlates with poor prognosis in multiple human cancers. Consistent with a direct requirement for IRF 4 for the enhanced sup- pressive functions, deletion of Irf 4 in Tregs enhanced antitumor immunity in a mouse model of cancer in vivo. We further identified a core network of immunosuppressive genes directly regulated by IRF4 in interaction with its molecular partner, BATF, thereby demonstrating that IRF4 instructs the suppressive activity of Tregs in human cancer.

\section{Results}

Treg heterogeneity in the TME and its relation to IRF4. To gain insight into $\mathrm{CD} 4^{+} \mathrm{T}$ cell phenotypes at the tumor site, we initially investigated $\mathrm{T}$ cells in a cohort of 53 patients with NSCLC (Supplemental Table 1 and ref. 24; supplemental material available online with this article; https://doi.org/10.1172/JCI130426DS1) with a 27-parameter polychromatic flow cytometry panel encompassing markers of memory and effector $\mathrm{T}$ cell differentiation, activation, metabolic activity, and exhaustion as well as Treg markers (Supplemental Table 2). We profiled tumors $(n=53)$, paired adjacent cancer-free lung tissues $(n=45)$, and peripheral blood samples (PB; $n=22$ ) of treatment-naive patients (Supplemental Table 1). We next used Uniform Manifold Approximation and Projection (UMAP), a dimensionality reduction visualization approach that preserves the local and the global structure of single-cell data, to simplify the visualization of marker coexpression in a 2D space (Figure 1, A and B). Overall, $\mathrm{CD} 4^{+} \mathrm{T}$ cells from the 3 body sites displayed a different single-cell profile (Figure 1A). Previously described dynamics aside (24), such as the loss of CD45RA', $\mathrm{CCR}^{+}$, and $\mathrm{CD} 27^{+}$(identifying naive and early memory cells) and the accumulation of $\mathrm{HLA}-\mathrm{DR}^{+}$activated and $\mathrm{PD} 1^{+} \mathrm{T}$ cells, we also noticed the accumulation of $\mathrm{CD} 25^{+} \mathrm{Foxp} 3^{+}$Tregs expressing the TF IRF4 in tumor versus adjacent lung and blood samples (Figure 1, B-D). Manual gating of flow cytometry data indicated that approximately $40 \%$ of $\mathrm{CD}^{2} 5^{+} \mathrm{Foxp}^{+}{ }^{+}$Tregs in tumors expressed IRF4, while the rest were IRF4- (Figure 1, C and D), suggesting Treg heterogeneity at the tumor site. In addition, the majority of conventional CD4 ${ }^{+} \mathrm{T}$ (Tconv) cells lacked IRF4 expression (Figure 1, C and D). Flow cytometric analysis also revealed that IRF4 ${ }^{+}$ Tregs retain increased expression of PD1, TIGIT, and TIM3 receptors and CD71 and CD98 metabolic markers compared with both IRF4- Tregs and Tconv cells in tumors (Figure 1E). These markers are generally upregulated with activation. In line with our results, reanalysis of published single-cell RNA-sequencing (scRNA-seq) data (25) of $\mathrm{CD}^{+}$tumor-infiltrating lymphocytes from patients with NSCLC identified IRF4 expression as largely confined to a subpopulation of CTLA $A^{\text {hi }}$ Tregs (subset 9-CTLA4; Figure 1F), while it was relatively lower in other cell subsets, including in nonactivated Tregs (8-FOXP3). CTLA4 $4^{h i}$ Tregs, which also express FOXP3, displayed an effector signature, including expression of CCR8, ICOS, TNFRSF4 (encoding OX-40), TNFRSF9 (encoding CD137/4-1BB), and the IRF4 transcriptional partner BATF (Figure 1G). We identified a similar subset of IRF4-expressing Tregs by scRNA-seq analysis of $\mathrm{CD}_{4} 5^{+}$cells infiltrating hepatocellular carcinoma (Supplemental Figure 1A and ref. 26). Notably, IRF4 expression correlated with multiple Treg genes but not with TFs related to other $\mathrm{Th}$ cell subsets in single $\mathrm{CD} 4^{+} \mathrm{T}$ cells isolated from melanomas (Supplemental Figure 1B and ref. 27). Thus, a common phenotypic and gene expression architecture defines tumor-infiltrating Tregs in multiple human cancers. 
A Gated on $\mathrm{CD}^{+} \mathrm{T}$ cells (tumor)

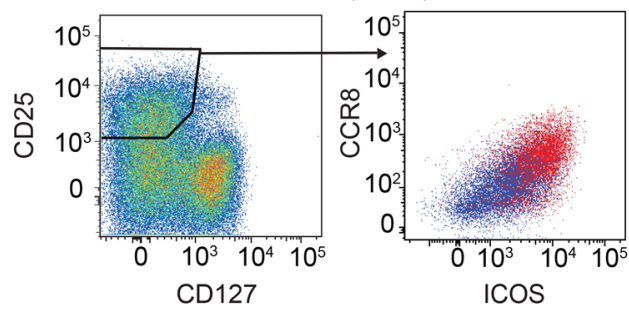

C

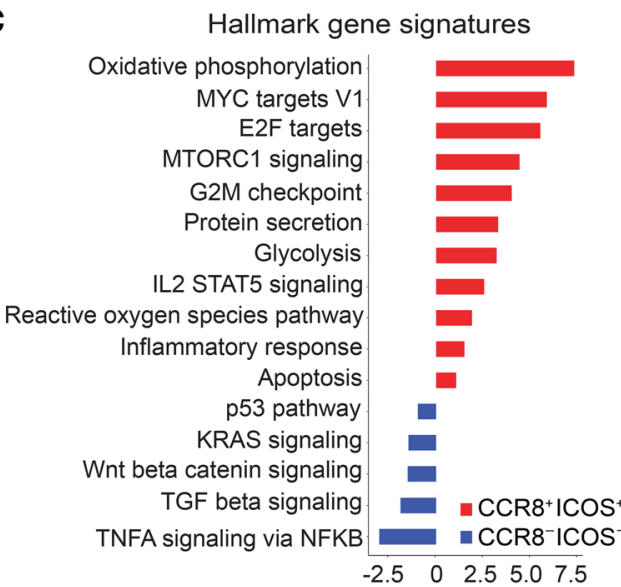

Normalized enrichment score (NES)
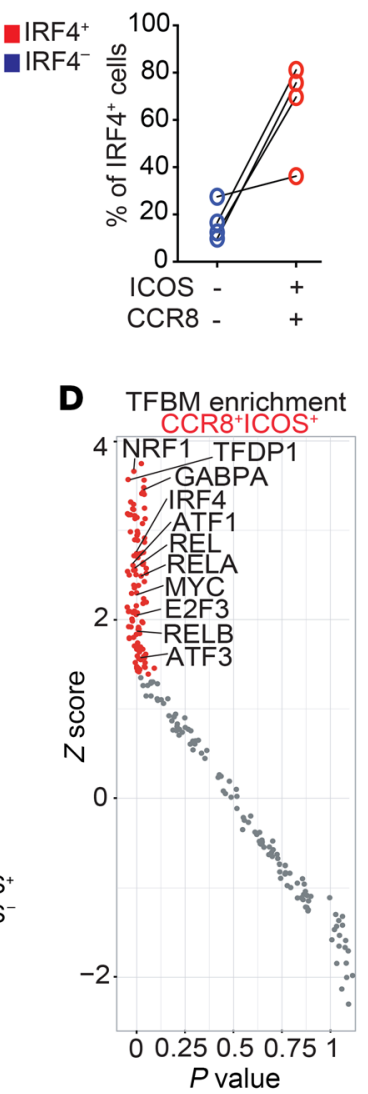

B

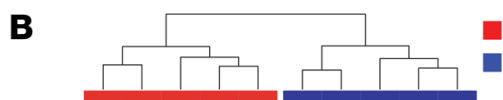

$\mathrm{CCR}^{+} \mathrm{ICOS}^{+}$

CCR8- ICOS
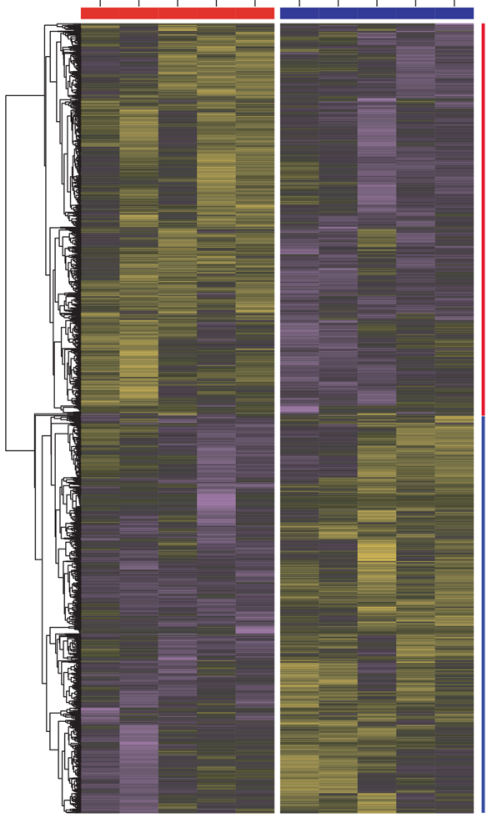

TNFRSF9 (4-1BB)

SOX4

TNFRSF4 (OX40)

ILIR2

TNFRSF8 (CD30)

IL2RA

$\angle A Y N$

CCR8

TNFRSF18 (GITR)

BATF

ICOS

ID3

IL 32

CTLA4

IKZF2 (Helios)

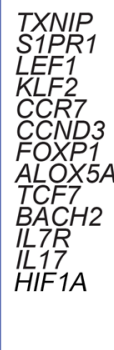

Color key

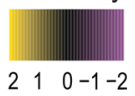

Row $Z$ score
E

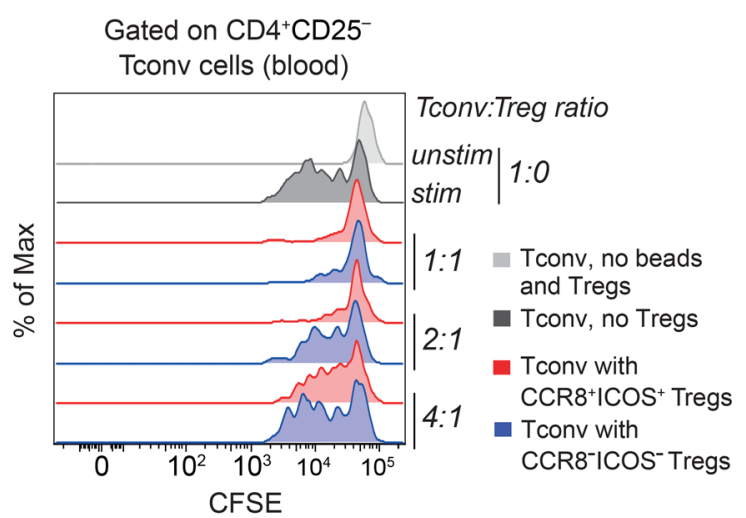

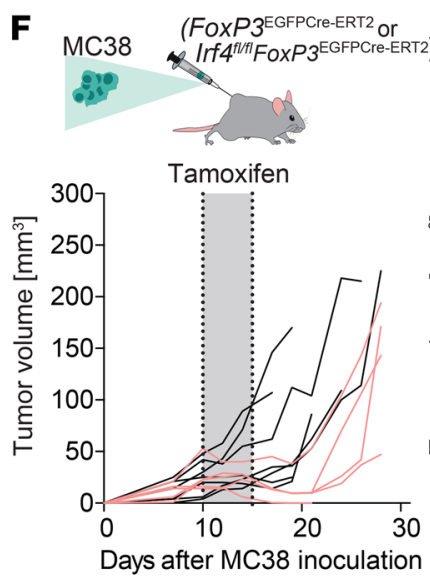

Tumor Tamoxifen

implant administration
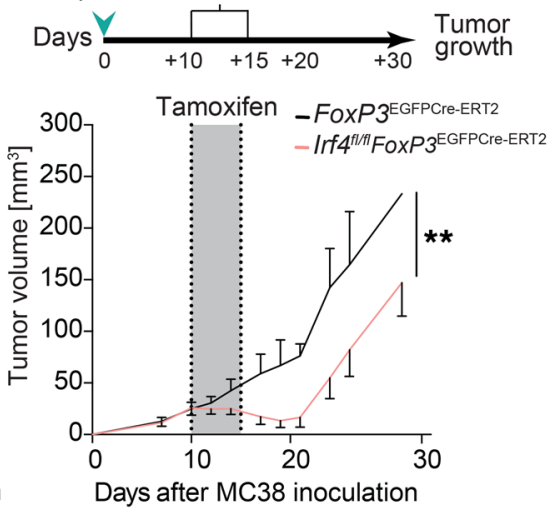

Figure 2. Transcriptional and functional profiling defines the effector and enhanced suppressive nature of IRF4+ Tregs. (A) Representative CCR8 and ICOS expression in tumor-infiltrating CD25 $5^{\text {hiCD1270 }}$ Treg subsets defined by IRF4 expression and t percentage of IRF4 expression in tumor-infiltrating Tregs gated as CCR8-ICOS- or CCR8 ${ }^{+}$ICOS $^{+}$. (B) Heatmap of differentially expressed genes (DEGs) in the FACS-sorted CCR8 ${ }^{+}$ICOS+ versus ICOS-CCR8- tumor-infiltrating Tregs, as obtained by RNA-seq (FDR $<0.05$ ). Selected DEGs are indicated. For some genes, protein names are indicated. (C) Hallmark gene sets (MsigDB; as obtained by GSEA) significantly enriched in cells sorted as in B. (D) Transcription factor binding motif (TFBM) enrichment analysis by pScan of RNA-seq data obtained as in B. Colored dots indicate significant hits. (E) CFSE-labeled CD4+ CD25- T (Tconv) cells dilution from a representative blood sample. Tconv cells were cocultured with Suppression Inspector MACSiBead beads and different ratios of intratumoral Treg subsets for 5 days. Data are

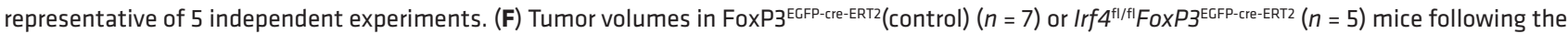
administration of tamoxifen. Tumor curves in individual mice and mean \pm SEM of the same cohort are shown. ${ }^{* *} P<0.01$, paired Student's $t$ test.

IRF4 expression defines effector Tregs with enhanced suppressive potential capable of promoting tumor growth in vivo. We further characterized IRF4 ${ }^{+}$and IRF4- Tregs by bulk RNA-seq. As the intranuclear localization of TFs precluded the isolation of viable cells based on IRF4 expression levels, we used a surrogate sur- face staining strategy for FACS-based isolation of Treg subsets. Bulk Tregs from tumors, defined as $\mathrm{CD} 25^{\text {hi }} \mathrm{CD} 127^{\mathrm{lo}}$, were further separated according to CCR8 and ICOS to identify IRF4 ${ }^{+}$and IRF4- Tregs (Figure 2A, Supplemental Figure 2A, and Methods). As expected, both of these subsets expressed high levels of FOXP3 
compared with Tconv cells (Supplemental Figure 2B) and their gene expression significantly overlapped with a tumor-infiltrating Treg signature, as obtained from a NSCLC scRNA-seq data set (ref. 25 and Supplemental Figure 2C), confirming the Treg identity of these subsets.

A multidimensional scaling plot of gene expression profiles showed that sorted intratumoral CCR8 ${ }^{+} \mathrm{ICOS}^{+}\left(\mathrm{IRF} 4^{+}\right)$and

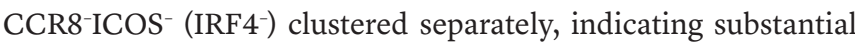
differences at the transcriptional level (Supplemental Figure 2D). Overall, we identified 2674 differentially expressed genes (DEGs; FDR $<0.05$; Supplemental Table 3). CCR ${ }^{+}$ICOS $^{+}$Tregs expressed high amounts of transcripts encoding molecules involved in effector differentiation (e.g., TNFRSF9, TNFRSF4, TNFRSF18, IL2RA), transcriptional regulation of effector Tregs $(B A T F)$, proliferation (MKI67), Treg identity (IKZF2, encoding the TF Helios [ref. 28]), costimulation (ICOS) and suppressive function (CTLA4). Additional transcripts included IL32, CCL22, and CX3CR1. By contrast, CCR8 ${ }^{-}$ICOS $^{-}$Tregs expressed high amounts of transcripts encoding molecules associated with early differentiation or quiescence, including CCR7, KLF2, LEF1, TCF7 (29), BACH2 (repressing effector programs to stabilize Treg-mediated immune homeostasis, ref. 30), and TXNIP (a negative regulator of AKT signaling and glycolytic metabolism) (Figure 2B and Supplemental Table 3). Gene set enrichment analysis (GSEA) further revealed that CCR8-ICOS Tregs were largely quiescent and displayed transcriptional signatures of Wnt $/ \beta$-catenin and TGF- $\beta$ signaling. In contrast, CCR $8^{+}$I$\mathrm{COS}^{+}$Tregs displayed enhanced signatures of metabolic activity, including oxidative phosphorylation and glycolysis; mTORC1-dependent activity, previously shown to favor murine Treg activation and prevention of autoimmunity (31); and reactive oxygen species metabolism, consistent with enhanced activation and/or mitochondrial respiration (Figure 2C, Supplemental Table 4, and ref. 32). To identify transcriptional regulators of $\mathrm{CCR}^{+} \mathrm{ICOS}^{+}$intratumoral Tregs, we performed computational analysis of TF-binding motif enrichment at the promoters $(-950,+50 \mathrm{bp}$ from the TSS) of DEGs obtained from bulk RNA-seq data. This analysis showed enrichment of binding motifs of TFs, including IRF4, predicted to be preferentially active in the CCR $8^{+} \mathrm{ICOS}^{+}$Treg subset (Figure 2D). We additionally identified NRF1, involved in antioxidant defense, lipid metabolism, and mitochondrial respiratory function (33); MYC, a master regulator of metabolic reprogramming in activated T cells (34); and the NF- $\kappa B$ family members REL, RELA, and RELB. In particular, RelA has been previously shown to regulate effector Treg activity in nonlymphoid tissues downstream of TNFRSFs (35). Thus, our transcriptional profiling revealed that intratumoral IRF4 $4^{+}$Tregs are highly activated compared with IRF4- Tregs and supported the notion that they have enhanced suppressive potential. To confirm this hypothesis, FACS-sorted

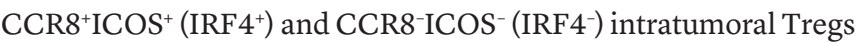
were further tested for their capacity to suppress proliferation of autologous $\mathrm{CD} 4^{+} \mathrm{CD} 25^{-}$Tconv cells in vitro (Figure $2 \mathrm{E}$ ). Both Treg subsets were effective in this regard at a 1:1 Tconv cell/Treg ratio, while only CCR ${ }^{+}$ICOS $^{+}$Tregs maintained substantial suppressive capacity at a 2:1 ratio (Figure $2 \mathrm{E}$ ).

IRF4 has been shown to promote effector Treg differentiation in peripheral tissues and to limit autoimmunity (20), but its importance in suppressing antitumor immune responses is unknown. To test the functional role of IRF4 in tumor-infiltrating Tregs, we used mice that allow tamoxifen-inducible deletion of IRF4 specifically in Tregs (Irf $\left.4^{\mathrm{E} / \mathrm{l} / \mathrm{F}} \mathrm{Foxp} 3^{\mathrm{EGFP}-\mathrm{cre}-\mathrm{ERT} 2}\right)$ and controls (Foxp3 $3^{\text {EGFP-cre-ERT2 }}$ ) (Figure 2F). Induced deletion of Irf4 in FoxP3 ${ }^{+}$ cells in MC38 tumor-bearing mice resulted in a significant delay in tumor growth (Figure $2 \mathrm{~F}$ ), indicating that IRF4+ $4^{+}$Tregs suppress antitumor immunity. To further test the Treg-intrinsic role of IRF4, we reconstituted lethally irradiated mice with a mix of congenically marked bone marrow from WT mice and mice with a $\mathrm{T}$ cell-specific deletion of IRF4 $\left(\operatorname{Irf} 4^{\mathrm{Il} / \mathrm{l} / \mathrm{C}} \mathrm{C} 4^{\mathrm{Cre}}\right)$. We also generated mixed control chimeras containing WT and $C d 4^{\text {Cre }}$ control bone marrow. Flow cytometric analysis of tumor-infiltrating Tregs in these chimeric mice showed severely impaired representation of IRF4-deficient Tregs at the tumor site but not in the spleen and lack of ICOS expression, a direct target of IRF4. In contrast, control chimeras showed similar contribution of Tregs at both sites and robust ICOS expression (Supplemental Figure 2, E and F).

IRF 4 and its partner BATF control a molecular program of effector Treg differentiation and suppression in tumors. Next, we formally tested the role of IRF4 and its transcriptional partner BATF in determining the features of tumor-infiltrating Tregs by integrating our data from human Tregs with gene expression profiles and epigenomic data from mouse Tregs (Supplemental Table 5). As mouse versus human gene regulation is difficult to infer due to species differences in noncoding regions, we first defined a conserved tumor-infiltrating Treg signature by integrating DEGs of CCR8 ${ }^{+}$ $\mathrm{ICOS}^{+}$versus CCR8 ${ }^{-} \mathrm{ICOS}^{-}$Tregs from Figure 2B and Supplemental Table 3 and DEGs of tumor-infiltrating Tregs versus spleen Tregs from a murine model (36), as outlined in Figure 3A. In total, we identified 382 transcripts that were specifically upregulated or downregulated in mouse and human tumor-infiltrating Tregs (Figure 3A and Supplemental Table 6). Next, we defined the transcriptional program that is dependent on IRF4 or BATF expression by performing RNA-seq and analysis of gene expression profiles of splenic Tregs from WT versus Irf4-/- mice. We also reanalyzed a published data set comparing WT versus Batf ${ }^{/-}$Tregs (37). Overall, this identified 1241 and 232 genes that were controlled by IRF 4 and BATF, respectively (Figure $3 \mathrm{~B}$ and Supplemental Table 5). GSEA revealed that many genes involved in lymphocyte activation, proliferation, and differentiation were under the joint control of IRF 4 and BATF; those involved in apoptosis were controlled by BATF alone; and those related to immunosuppression, i.e., IL-37and IL-10-dependent signaling pathways, were under the control of IRF4 alone (Supplemental Figure 3B). A relevant proportion (94 genes; $24.6 \%$ ) of the conserved tumor Treg signature between humans and mice was controlled by IRF4 and/or BATF (Figure 3B; IRF 4 and BATF DEGs vs. tumor-infiltrating Treg signature: $P=1.1$ $\times 10^{-14}$ and $P=3.3 \times 10^{-18}$, respectively; hypergeometric test; data not shown). We further made use of our ChIP deep-sequencing (ChIP-seq) data to assess IRF4 occupancy at the loci of interest (Supplemental Table 7) and thus define a direct role of this TF in controlling gene expression in tumor-infiltrating Tregs. Supplemental Figure 3B shows the distribution of regions in the genome that are bound directly by IRF4 according to their distance from transcriptional start sites (TSSs) (see also Supplemental Table 7). Despite a small fraction mapped in promoter regions $(0-1 \mathrm{~Kb})$, the majority of IRF4-bound sites mapped at 10 to $100 \mathrm{~Kb}$ dis- 
A

$\mathrm{CCR}^{+} \mathrm{ICOS}^{+}$vs

CCR8 ${ }^{-}$ICOS $^{-}$DEGs

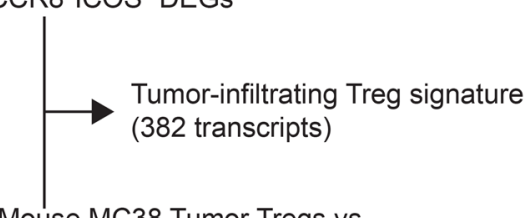

Mouse MC38 Tumor Tregs vs

Spleen Tregs (ref. 36)
B

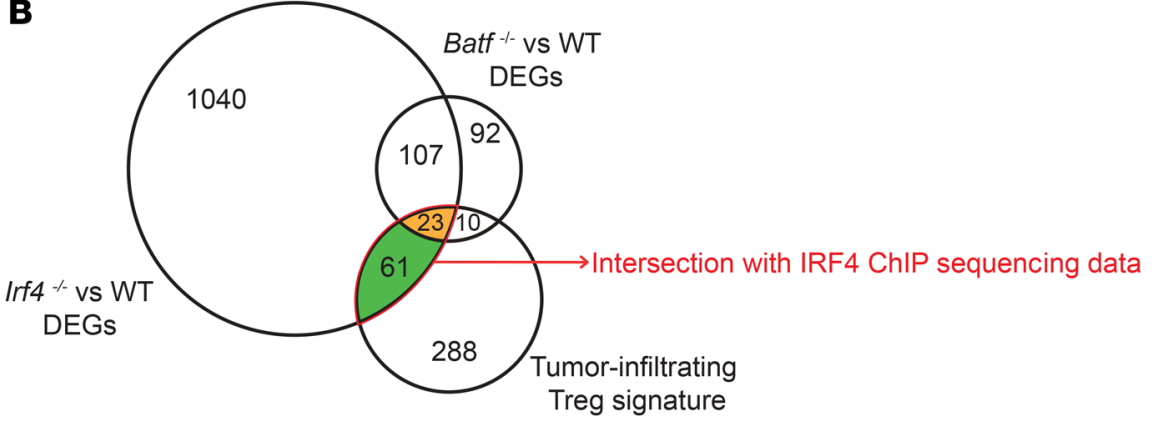

C $\quad$ IRF4

dependent genes
IRF4 ChIP sequencing

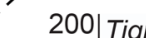

200| Tigit

0

Tigit
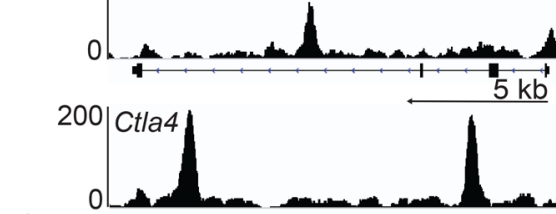

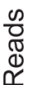

$200 \mid$ Tnfrsf1b
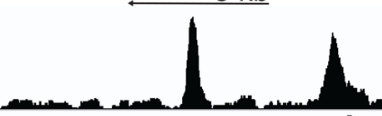

$5 \mathrm{~kb}$
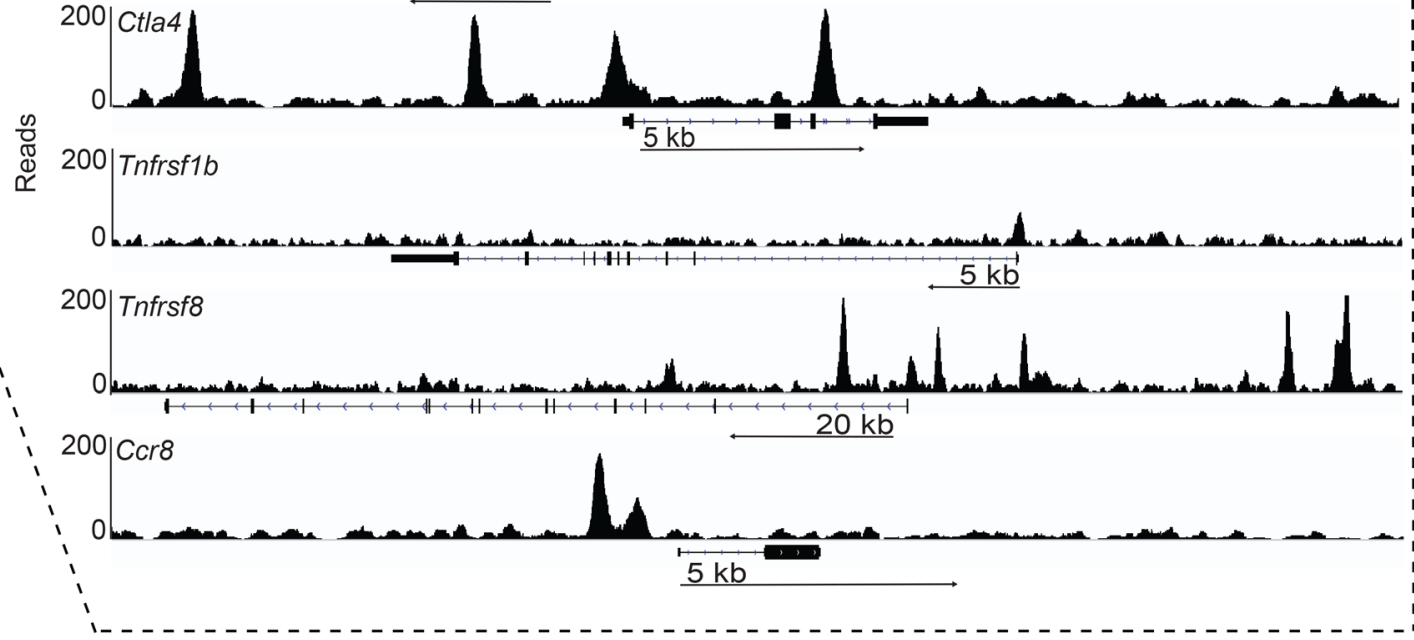

IRF4 ChIP sequencing

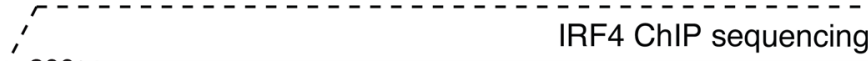

Rrm2

Aurkb

IRF4 \& BATF dependent genes

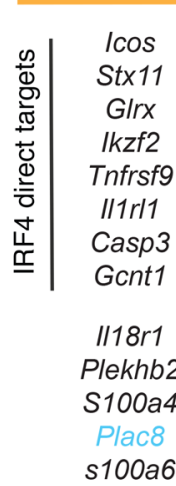

, $200 \mid / \cos$

i

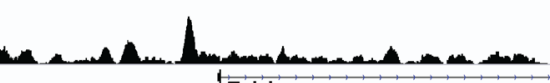

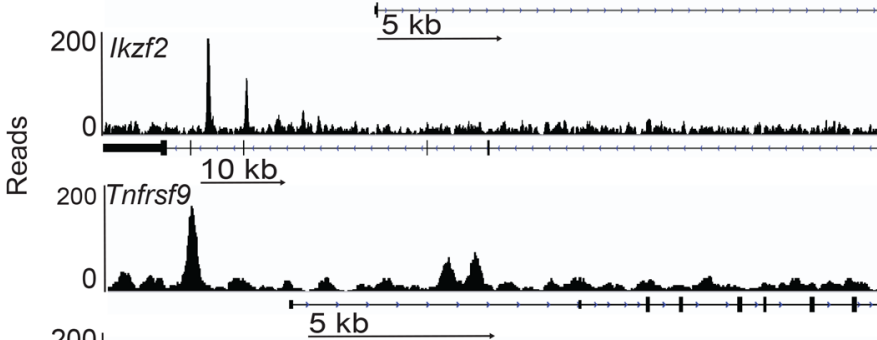

$200 \mid / 11 r / 1$

$---i$

0

$5 \mathrm{~kb}$ 
Figure 3. Irf4 and its partner Baft directly and indirectly control a molecular program of effector Treg differentiation and suppression. (A) Identification of a shared gene expression signature between tumor-infiltrating human CCR8 ${ }^{+}$ICOS ${ }^{+}$Tregs versus CCR8-ICOS- Tregs and murine Tregs (36). (B) Venn diagram of the number of genes of the tumor-specific Treg signature obtained as in $\mathbf{A}$ that are differentially expressed in splenic Tregs from Batf ${ }^{-1-}$ and $/ \mathrm{rf}^{-1-}$ mice. Genes controlled only by Batf $(n=10)$ were of limited interest and thus not further investigated. (C) List of tumor-infiltrating Treg genes that are dependent on the expression of Irf4 or Irf4 and Batf. All genes are induced, except for Plac8, which is repressed (indicated in light blue). Those genes directly controlled by IRF4 binding to the genome, as obtained from ChIP-seq analysis of murine Tregs, are highlighted. Genomic binding of Irf4 to the DNA for selected genes is depicted.

tance from the TSS, suggesting regulation of gene expression at enhancer regions. Tumor-infiltrating Treg genes dependent on IRF4 included several Tnfr family members involved in effector Treg differentiation, such as Tnfrsf1b, Tnfrsf8, Tnfrsf18, and Tnfrsf9 (the latter also dependent on Batf expression) (35); chemokine receptors, such as Ccr8, Cxcr3, and Ccr5, likely involved in the localization of Tregs to tumors or to the lung and expressed by murine effector Tregs (38); and Zbtb32, Mki67, Map2k3, Kif23, Rrm2, and Aurkb, previously linked to cell cycle in other cell types and likely contributing to Treg proliferation (Figure 3C). Instead, Icos, Ikzf2 (encoding Helios), and Il1rl1 (also known as ST2 or IL-33 receptor) were dependent on both Irf4 and Batf. Thus, Irf4 and Batf directly and indirectly control a program of effector Treg differentiation and immunosuppression in cancer.

scRNA-seq-guided high-dimensional flow cytometry profiling reveals that $\mathrm{CCR}^{+} \mathrm{ICOS}^{+}\left(\mathrm{IRF}^{+}\right)$effector Tregs associate with multiple exhaustion traits of $T$ cells. To further characterize the $\mathrm{T}$ cell phenotypic landscape associated with Treg subsets in patients with NSCLC cancer in an unbiased manner, we designed a second high-dimensional flow cytometry panel guided by results obtained with scRNA-seq (25) and bulk RNA-seq (Figure 2 and Supplemental Table 2). We preferred markers identified by fluorochrome-conjugated antibodies providing a high signal-to-noise ratio (e.g., CCR8 and ICOS instead of IRF4) so as to enhance the identification of subsets by the clustering algorithm, as recently suggested (39). We profiled millions of single cells from tumors $(n=45)$, paired adjacent cancer-free lung tissues $(n=23)$, and peripheral blood samples $(n=23)$ of treatment-naive patients (Supplemental Table 1$)$. A schematic representation of the analysis pipeline is shown in Figure 4A. By applying PhenoGraph, a computational algorithm capable of clustering single cells without bias according to the relative expression of these molecules in the multidimensional space (40), we identified 14 different $\mathrm{CD}^{+}$and 15 different $\mathrm{CD}^{+} \mathrm{T}$ cell clusters and defined their abundance as a percentage of total $\mathrm{CD} 4^{+}$or $\mathrm{CD}^{+} \mathrm{T}$ cells in each sample type (Figure $4 \mathrm{~B}$ ). In addition, for this panel of markers, UMAP of single-cell distributions and principal component analysis (PCA) of cluster abundance in the different samples clearly distinguished $\mathrm{T}$ cells from the peripheral blood, lung tissues, and tumors (Supplemental Figure 4, A and B), indicating that different sites have highly distinct $\mathrm{T}$ cell profiles. We next calculated the integrated median fluorescence intensity (iMFI) values of each marker in each PhenoGraph cluster so as to obtain information on cluster identity (Figure 4B, the Methods, and ref. 39). Metaclustering of PhenoGraph clusters and markers grouped subpopulations with similar immunophenotypes. Similar to that in previous findings (24), we documented the loss of subsets of naive $\left(\mathrm{CD}^{+}, \mathrm{C} 2 ; \mathrm{CD}^{+}, \mathrm{C} 12\right)$ and cytotoxic $\left(\mathrm{CD}^{+}, \mathrm{C} 7 ; \mathrm{CD}^{+}, \mathrm{C} 1, \mathrm{C} 9, \mathrm{C} 2\right)$ $\mathrm{T}$ cells and the accumulation of exhausted $\mathrm{T}$ cells $(\mathrm{CD} 8, \mathrm{C5})$ in tumors compared with the blood or the adjacent lung tissue (Figure $4 \mathrm{~B})$. Moreover, in line with data shown in Figure 1, we revealed the increased presence of $\mathrm{CCR} 8^{+} \mathrm{ICOS}^{+}\left(\mathrm{IRF} 4^{+}\right)$activated Tregs $\left(\mathrm{CD} 4^{+}\right.$, C6) in tumors. Next, we investigated the association of these cells with specific $\mathrm{T}$ cell subsets in the TME by performing a Pearson correlation analysis of the abundance of $\mathrm{CD} 4^{+}$and $\mathrm{CD}^{+} \mathrm{T}$ cell clusters as identified by PhenoGraph in 45 patients (Figure $4 \mathrm{C}$ ). Notably, intratumoral $\mathrm{CCR}^{+} \mathrm{ICOS}^{+}$Tregs correlated with $\mathrm{CD} 4^{+}$ (C13) and $\mathrm{CD}^{+}$(C5 and C15) T cells with features of exhaustion (i.e., expressing PD1, TIM3, and TIGIT in different combinations and intensities). At the same time, $\mathrm{CCR} 8^{+} \mathrm{ICOS}^{+}$Tregs negatively correlated with cytotoxic $\mathrm{CD} 8^{+} \mathrm{T}$ cells (C2, coexpressing GZMB and GNLY and thus armed for rapid effector functions) and with clusters of $\mathrm{T}$ cells bearing a central memory $\mathrm{T}$ phenotype (CD4 ${ }^{+}$, $\mathrm{C} 11$ and $\left.\mathrm{CD}^{+}, \mathrm{C} 14\right)$. In summary, high-dimensional single-cell profiling identifies a $\mathrm{T}$ cell signature with increased frequencies of $\mathrm{CCR}^{+} \mathrm{ICOS}^{+}$effector Tregs associated with $\mathrm{T}$ cell exhaustion.

$\mathrm{CCR}^{+} \mathrm{ICOS}^{+}\left(\mathrm{IRF} 4^{+}\right)$effector Tregs define a signature of disease progression in NSCLC. We finally tested whether a phenotypic landscape of $\mathrm{T}$ cells involving IRF $4^{+}$effector Tregs could be found to be different in patients with cancer with different prognoses. First, a subset of our cohort of patients $(n=25)$ was subdivided in 2 groups according to the median distribution of the maximum standardized value of fluorodeoxyglucose uptake (SUVmax), a PET indicator of tumor glycolysis and aggressiveness. PCA revealed a bimodal separation of the SUVmax ${ }^{\mathrm{lo}}$ and SUVmax ${ }^{\text {hi }}$ groups of patients according to the relative frequencies of $\mathrm{CD}^{+}$and $\mathrm{CD} 8^{+}$ PhenoGraph clusters (Figure 5A, left), indicating that they display a different $\mathrm{T}$ cell profile as a whole. Analysis of PCA loadings identified clusters contributing the most to such distribution (Figure $5 \mathrm{~A}$, right). Specifically, SUVmax ${ }^{\text {hi }}$ patients harbored increased frequencies of CCR8 ${ }^{+} \mathrm{ICOS}^{+}$Tregs (C6), subsets of $\mathrm{CD}^{+}$(C5 and $\mathrm{C} 15)$, and $\mathrm{CD}^{+} \mathrm{T}$ cells (C5) with traits of exhaustion or memory $\mathrm{T}$ cells expressing GZMK, EOMES (contributing to T cell dysfunction in humans [ref. 41]), and PD1 (CD8 $\left.{ }^{+}, \mathrm{C} 3, \mathrm{C} 7\right)$, while SUVmax ${ }^{\text {lo }}$ patients harbored increased frequencies of $\mathrm{CCR} 5^{+}$clusters $\left(\mathrm{CD} 8^{+}\right.$, $\mathrm{C} 4, \mathrm{C} 6$ and $\left.\mathrm{CD}^{+}, \mathrm{C} 3, \mathrm{C} 4\right)$ as well as terminal effector, cytotoxic $\mathrm{T}$ cells $\left(\mathrm{CD}^{+}, \mathrm{C} 1\right.$ and $\mathrm{CD} 4^{+}, \mathrm{C} 7$ ) (Figure $5 \mathrm{~A}$ ). We observed similar trends when considering a parameter of disease progression, i.e., the international TNM classification, according to which CCR8 ${ }^{+}$ $\mathrm{ICOS}^{+}$Tregs $\left(\mathrm{CD}^{+}, \mathrm{C} 6\right)$ as well as T cells featuring exhaustion/ activation markers $\left(\mathrm{CD}^{+}, \mathrm{C} 5\right.$ and $\left.\mathrm{C} 15\right)$ were more abundant in patients with advanced pathological stage II and III (Figure 5B).

Infiltration of $\mathrm{CD}^{+} \mathrm{T}$ cells is a predictor of good prognosis in multiple types of cancer (42). We thus investigated disease-free survival (DFS) of our patients according to the Treg/CD8 ${ }^{+} \mathrm{T}$ cell ratio (as determined by their frequency among $\mathrm{CD}^{+}$) at the tumors site and found that a higher ratio was significantly associated with early tumor relapse (Figure $5 \mathrm{C}$ ). As predicted by their superior suppressive activity, this was due to the contribution of $\mathrm{CCR}^{+} \mathrm{ICOS}^{+}$, but not of CCR8 ${ }^{-}$ICOS $^{-}$, Tregs (Figure $5 \mathrm{C}$ ). We next validated our findings in larger cohorts of individuals, such as those from The Cancer Genome Atlas (TCGA). In these, the 
A

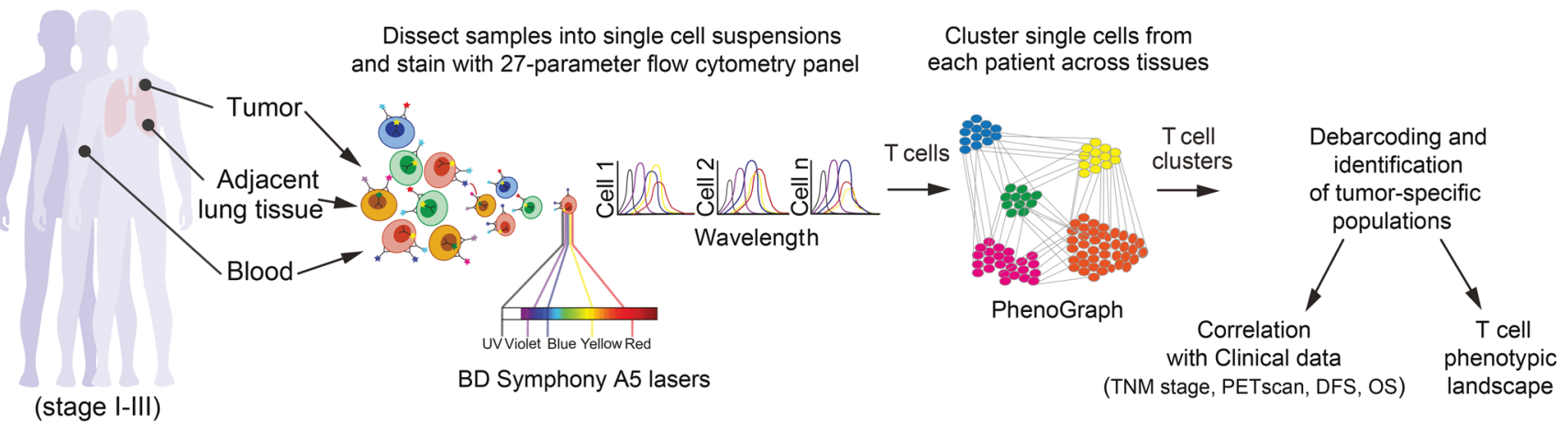

NSCLC patients
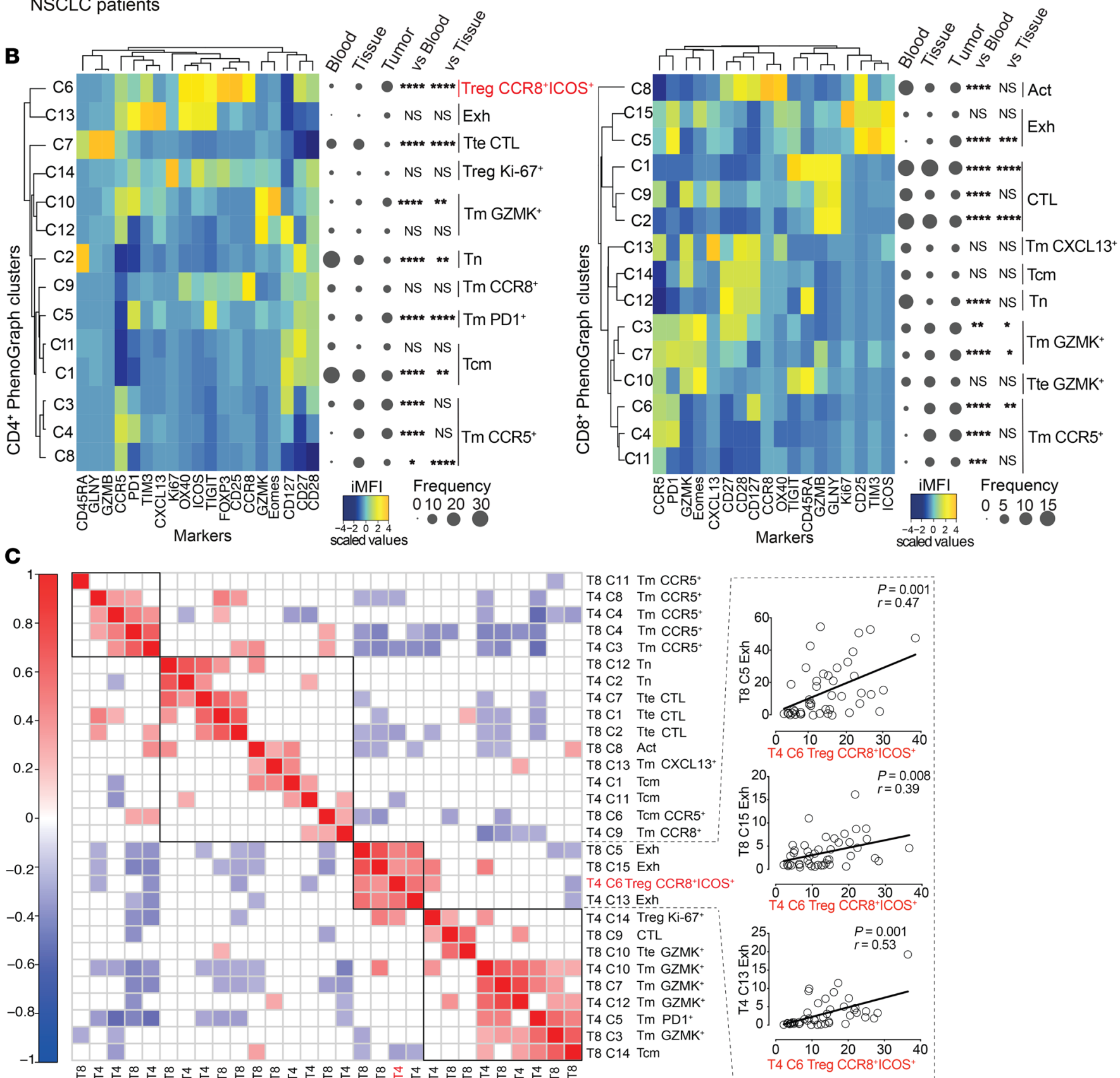

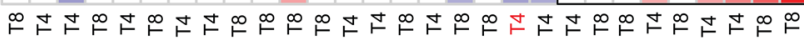

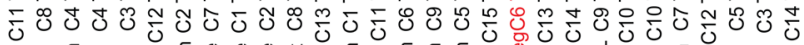

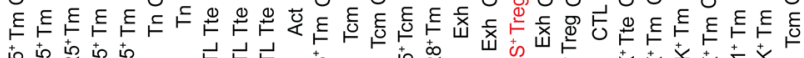

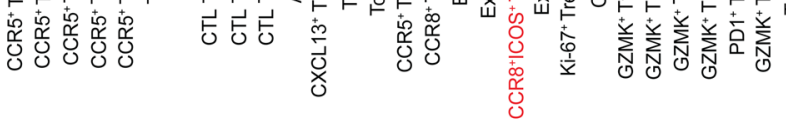


Figure 4. Abundance of CCR8 ${ }^{+} \mathrm{ICOS}^{+}$intratumoral Tregs is associated with multiple features of T cell exhaustion. (A) Experimental workflow. (B) Heatmaps of the relative expression, depicted as integrated MFI (iMFI: $\mathrm{MFI} \times$ percentage of antigen expression) of markers (columns) in discrete $\mathrm{CD}^{+}$(left) and CD8+ (right) Phenograph clusters (rows). Tm, memory; Tcm, central memory; Tn, naive; Exh, exhausted; Act, activated; CTL, cytotoxic T lymphocyte. Tte, terminal effector. Data are further metaclustered to group subpopulations with similar immune-phenotypes. The median frequency of each PhenoGraph cluster in the different compartments is depicted by using Balloon plots. ${ }^{*} P<0.05$; ${ }^{* *} P<0.01$; ${ }^{* *} P<0.001$; ${ }^{* * *} P$ $<0.0001$, tumor versus blood or versus normal tissue samples; 2-way ANOVA with Bonferroni's post hoc test. (C) Correlogram showing Pearson correlation between frequencies of $\mathrm{CD}^{+}(\mathrm{T} 4)$ and $\mathrm{CD} 8^{+}$(T8) PhenoGraph clusters in tumor samples from 45 patients with early-stage (I-III) nonsmall-cell lung cancer (NSCLC). Nonsignificant correlations ( $P$ value $>0.05$ ) were left blank.

degree of infiltration of specific subpopulations was determined by enrichment of transcriptional signatures (see Methods). Similar to results from our NSCLC cohort, we found that a higher CCR $8^{+} \mathrm{I}-$ $\mathrm{COS}^{+} \mathrm{Treg} / \mathrm{CD}^{+} \mathrm{T}$ cell signature in bulk RNA-seq data from primary biopsies was associated both with worse DFS and overall survival in lung adenocarcinoma (Figure 5D and ref. 43), hepatocellular carcinoma (Supplemental Figure 5A and ref. 44), and melanoma (Supplemental Figure 5B), thereby underlying a common biology of Treg-mediated suppression of antitumor immunity in multiple human cancers.

\section{Discussion}

Human tumors are often infiltrated by large numbers of $\mathrm{CD} 4^{+}$ Tregs that display a highly activated phenotype and enhanced suppressive capacity compared with those present in the peripheral and the adjacent tumor-free tissues $(15,16)$. We have now shown that the transcriptional program associated with such activation and suppression in the TME is driven by the TF IRF4 in combination with its molecular partner BATF. Signals downstream of the TCR are likely involved in IRF4 induction (45), possibly suggesting that human Tregs suppress antitumor immunity in an antigen-specific way.

IRF4, previously linked to effector Treg differentiation in murine tissues in physiology (20), is now shown to control the formation of ICOS ${ }^{+}$effector Tregs in the TME that, in turn, favor tumor growth in a mouse model of cancer. IRF 4 controls gene expression of tumor-infiltrating Tregs both directly, by binding gene promoters or distal regulatory regions, and indirectly, by inducing the expression of additional transcriptional regulators, such as IKZF2 (Helios), which is required for Treg stability (28). IRF4 alone binds DNA poorly; however, binding is increased when IRF4 is part of a macromolecular complex involving AP-1 family members BATF, JUN, JUNB, or JUND, which recognize DNA motifs known as AP-1/IRF composite elements (AICEs) (21, 46). Although the regions bound by these TFs are largely overlapping, IRF4 and BATF also bind unique regions (21), which possibly explains why their deficiency has differential, specific effects on gene expression. We did not formally test whether BATF is responsible for the expression of specific genes by direct binding; however, additional computational investigations identified AICE motifs in the proximity of genes that are linked to effector Treg differentiation and function, including Ccr8, Icos, Ctla4, Cxcr3, Il12rb1, Tnfrsflb, and Tnfrsf8 (data not shown). In relation to this, JunB has recently been shown to control murine effector Treg differentiation in the lung and colon (47), while additional TFs, such as STAT3, may physically interact with c-Jun (48). STAT3 mRNA is upregulated and the STAT3 binding motif is enriched in IRF $4^{+}$ Tregs compared with IRF4- Tregs (data not shown), which overall suggests that a complex range of molecular interactions is cooperating to shape the effector differentiation and enhanced suppressive activity of intratumoral Tregs.

Our study reveals an additional important aspect, namely that effector differentiation is not a feature of all intratumoral Tregs. Rather, a subset of these cells with increased expression of IRF4 is preferentially expanded in lung, liver, and melanoma tumors compared with the adjacent tumor-free tissues and peripheral blood, as revealed by scRNA-seq. These IRF $4^{+}$Tregs express high amounts of molecules associated with enhanced immunosuppression, have increased metabolic demand, and are phenotypically and transcriptionally distinct from the more quiescent IRF4- Tregs. Whereas in humans approximately $40 \%$ of Tregs express IRF4, as detected by flow cytometry, deletion of Irf 4 in mice results in the near-complete loss of Tregs in tumors, which possibly indicates that low levels of IRF 4 are also present in CCR8-ICOS- Tregs. An alternative hypothesis is that slow tumor development, as seen in humans, results in a balanced infiltration of fewer and more activated Tregs. In line with this, approximately $40 \%$ of Tregs infiltrating lung adenocarcinoma forming in the K-ras ${ }^{\mathrm{G} 12 \mathrm{D}}$ autochthonous model (which better recapitulates of the physiology of lung adenocarcinoma) express the IL-33 receptor ST2 (encoded by Illrl1) (49), a direct genomic target of IRF4 (Figure 3C). Similarly, a recent human study found that approximately $50 \%$ of breast cancer-infiltrating Tregs express CCR8 (50), thereby corroborating our results of Treg heterogeneity in the TME. Despite not reaching the capability of scRNA-seq in terms of number of parameters being measured, the high-dimensional flow cytometry used here investigates $\mathrm{T}$ cell phenotypes as a whole in a large cohort of patients compared with those recently investigated by scRNA-seq (25) and is thus capable of further defining the relationships between Treg subsets and other $\mathrm{T}$ cell populations within the TME. In this way, we revealed that IRF4 $4^{+}$ Tregs positively correlate with multiple subsets of exhausted and activated $\mathrm{CD} 4^{+}$and $\mathrm{CD} 8^{+} \mathrm{T}$ cells and their abundance is associated both with relapse and poor overall survival in multiple human cancers. Therefore, deep immunophenotyping with improved and high-throughput single-cell technologies can pinpoint those subsets associated with slower tumor growth and favorable antitumor responses with enhanced precision. We anticipate that the simple addition of an effector Treg marker (e.g., IRF4, ICOS, or CCR8 or a TNFR superfamily member) to FoxP3 staining, as done by immunohistochemistry in immunoscore approaches (51), will improve the definition of patients with improved prognosis.

The identification of $\mathrm{CD} 4^{+}$Treg heterogeneity in the TME and, as a consequence, of a molecular program mastering the differentiation of those Tregs with enhanced suppressive capacity offers potentially novel opportunities to reverse immunosuppression while favoring antitumor immune responses. Depletion of Tregs has been tested in a number of preclinical approaches to promote 

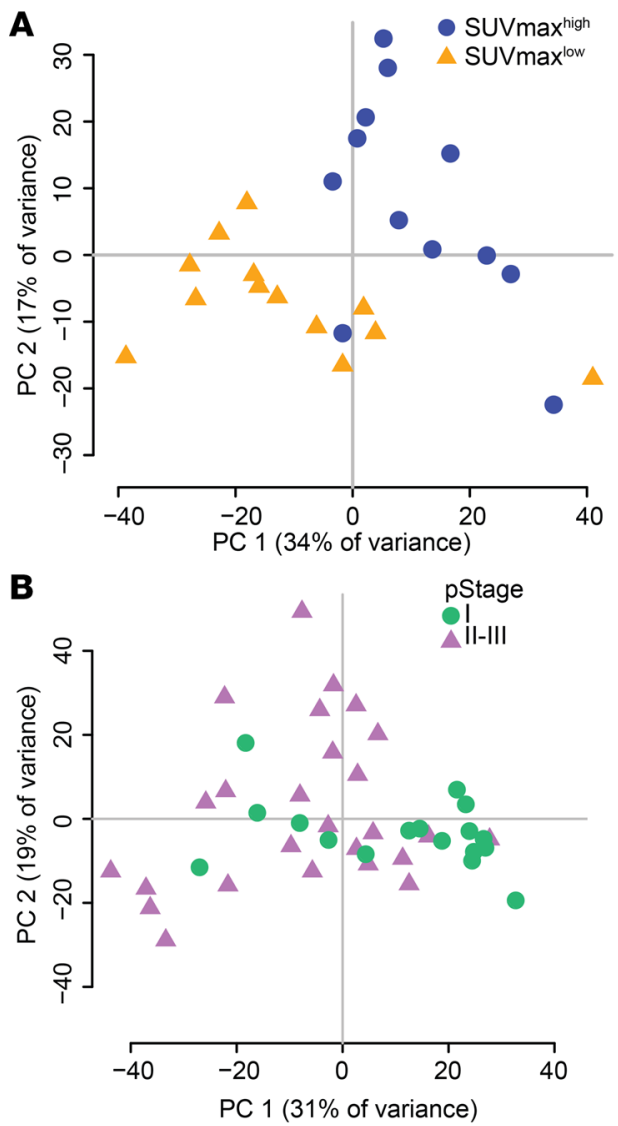
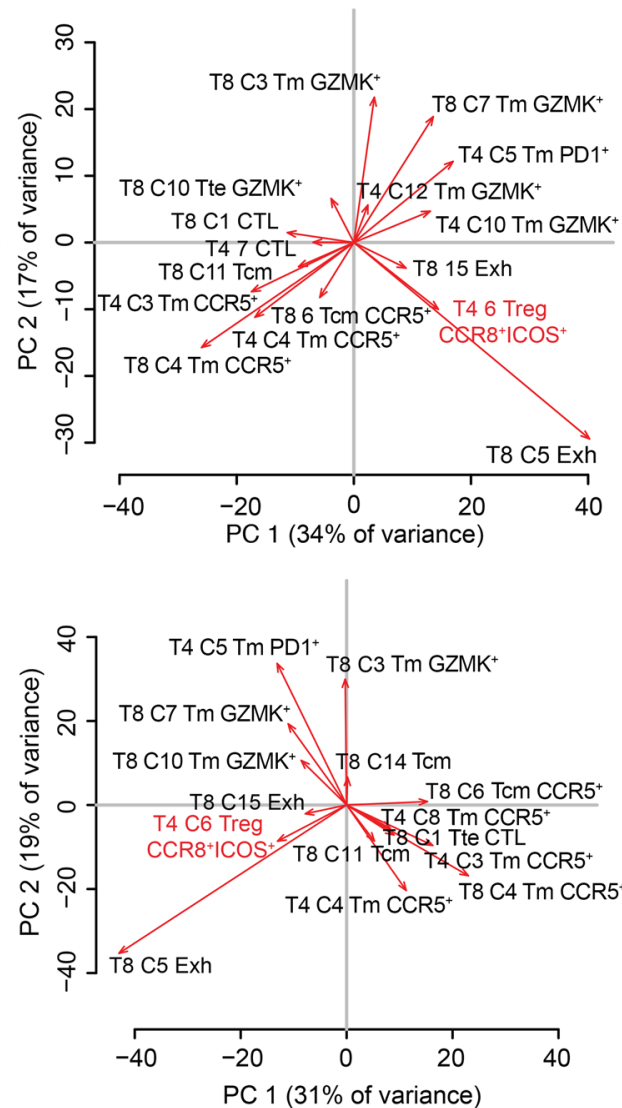
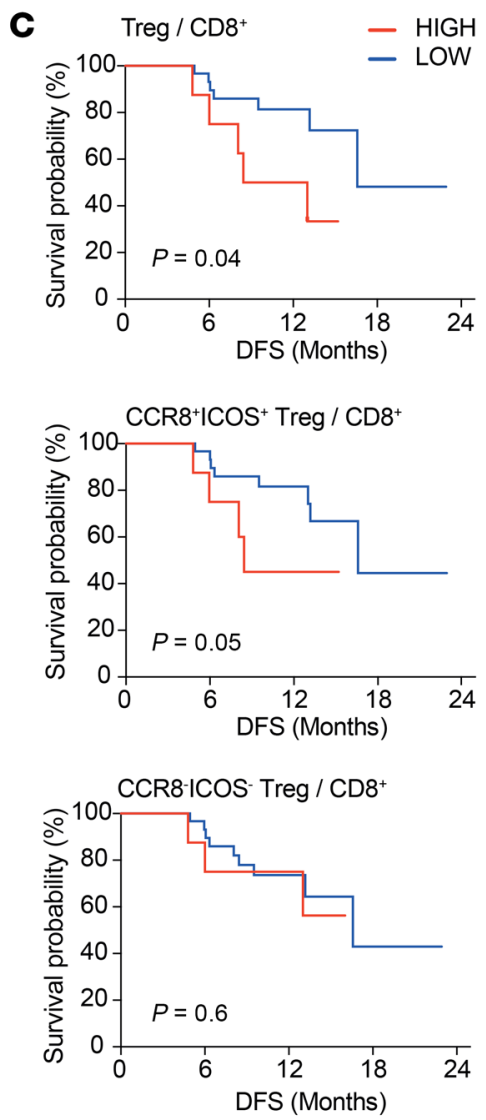
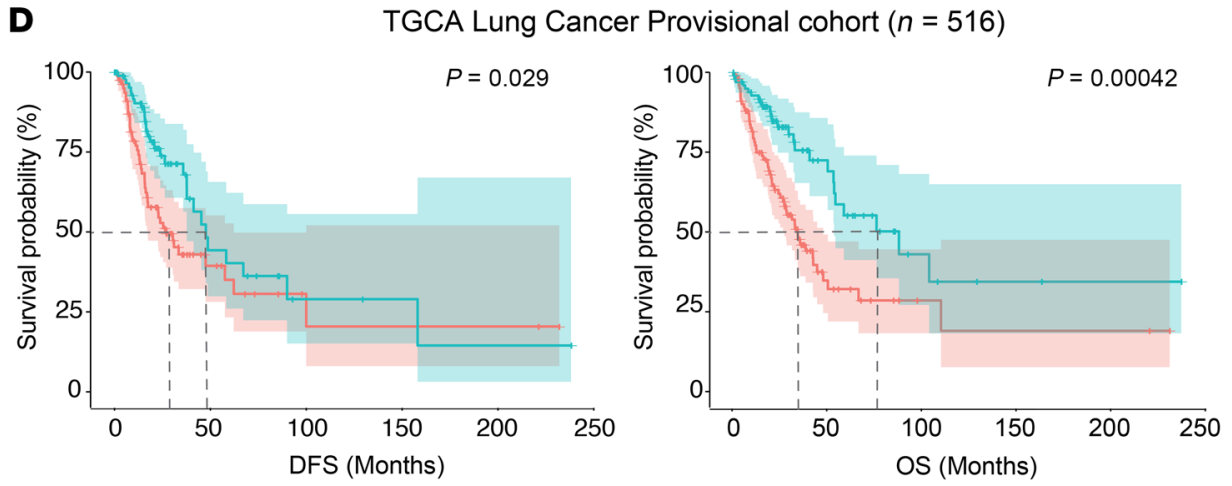

CCR8 ${ }^{+} \mathrm{ICOS}^{+}$Treg / CD8 ${ }^{+}$ signature enrichment

$+\mathrm{HIGH}$

+ LOW

Figure 5. CCR8 ${ }^{+}$ICOS$^{+}$Tregs define a signature of disease progression in NSCLC. (A) Left: Principal component analysis (PCA) plot showing the distribution of patients $(n=48)$ according to the frequency of $C D 4^{+}$and $C D 8^{+}$PhenoGraph clusters in each patient (Tm, memory; Tcm, central memory). Patients were classified according to pathological stage (pStage) I, II, or III of the International TNM classification. Right: PCA loading plot of PhenoGraph clusters most contributing to the PCA output on the left. (B) Left: PCA plot showing the distribution of patients $(n=26)$ according to the frequency of CD4+ and CD8 ${ }^{+}$ PhenoGraph clusters in each patient. The cohort was subdivided in 2 groups according to the median distribution of maximum standardized uptake value (SUVmax). Right: PCA loading plot as in A. (C) Kaplan-Meier progression-free survival curves according to the intratumoral frequencies of Tregs subsets over CD8+ T cells in each patient $(n=38)$. The cohort was subdivided in 2 groups according to the percentile rank (set at 0.8$)$. The $P$ value was calculated by Gehan Breslow-Wilcoxon test. (D) Kaplan-Meier disease-free survival (DFS) and overall survival (OS) curves in the TCGA lung adenocarcinoma (LUAD) lung cancer cohort $\left(n=516\right.$ ). Patients were grouped by percentile rank (set at 0.8 ) according to the enrichment of the CCR $8^{+}$ICOS ${ }^{+}$bulk Treg signature (as obtained in Figure 2B) as relative to the $\mathrm{CD} 8^{+} \mathrm{T}$ cell signature. + indicates censored observations. $P$ values were calculated by multivariate Cox regression. Dotted lines indicate the time at which $50 \%$ of the cohort was still free of the event.

antitumor responses (11-14); however, novel strategies interfering selectively with the activated effector Treg state are emerging as promising tools to boost effective antitumor immunity without resulting in overt autoimmunity due to the loss of peripheral tolerance $(14,52)$. We expect that targeting those signals leading to IRF 4 activation or downstream IRF4-dependent Treg activation would result in a similar scenario. This approach may be widely applicable, as our data show that IRF4-driven effector Treg differentiation is common to at least 3 human tumor types, i.e., lung cancer, hepatocellular carcinoma, and melanoma. Further definition of the 
molecular network orchestrating the suppressive capacity of intratumoral Tregs and, most importantly, the identification of specific players that are not active in antitumor-infiltrating lymphocytes is anticipated to benefit cancer immunotherapy strategies.

\section{Methods}

Study design. The characteristics of the patients and of the samples used in this study as well as the procedures of cell isolation have been described previously (24). Details on the patients' characteristics included in this manuscript are further indicated in Supplemental Table 1. Information on the pathological stage, determined by an institutional pathologist (some patients were restaged as III following examination of the tumor), was available for all patients, while results of the preoperative FDG PET-CT scan were available for 25 patients. Details on obtainment of PET scans have been described previously (24).

Polychromatic flow cytometry and cell sorting. Antibodies used in the study are listed in Supplemental Table 2. Flow cytometry procedures for high-dimensional single-cell panel development have been described previously $(24,39)$. Additional panels used for further characterizing the $\mathrm{IRF} 4^{+} \mathrm{CD} 4^{+}$cell subset are listed in Supplemental Table 2. All data were acquired on a FACS Symphony A5 flow cytometer (BD Biosciences) equipped with 5 lasers (UV, $350 \mathrm{~nm}$; violet, $405 \mathrm{~nm}$; blue, $488 \mathrm{~nm}$; yellow/green, $561 \mathrm{~nm}$; red, $640 \mathrm{~nm}$; all tuned at $100 \mathrm{~mW}$, except for UV, which tuned at $60 \mathrm{~mW}$ ) and the capability of detecting 30 parameters. Flow cytometry data were compensated in FlowJo by using single stained controls (BD Compbeads incubated with fluorescently conjugated antibodies), as described previously (53). CCR8 ${ }^{+}$I$\mathrm{COS}^{+}$and CCR8- $\mathrm{ICOS}^{-}$Tregs, pregated as CD4 ${ }^{+}$Aqua $^{-} \mathrm{CD} 25^{+} \mathrm{CD} 127^{-}$, were isolated from tumor samples with a FACSAria cell sorter (BD Biosciences). CCR 8 and ICOS proved to be the best combination over other markers to isolate Treg subsets with differential IRF4 expression (data not shown).

Suppression of $T$ cell proliferation by Tregs. Live (Aqua-) $\mathrm{CD} 4^{+} \mathrm{CD} 25$ Tconv cells were isolated from patients' blood samples with a FACSAria cell sorter and stained with a CellTrace CFSE kit (final concentration: $2 \mu \mathrm{M}$; Thermo Fisher Scientific) according to the manufacturer's protocol. Subsequently, cells were plated in R10 U-bottom 96-well plates (10,000 cells/well) and stimulated with human Treg Suppression Inspector beads (Miltenyi Biotec) for 5 days at $37^{\circ} \mathrm{C}$. Tconv cells cultured alone, in the absence of bead stimulation, were used as nonproliferating, negative control. CCR8 ${ }^{+} \mathrm{ICOS}^{+}$and CCR8 ${ }^{-} \mathrm{ICOS}^{-}$Tregs were FACS sorted from tumors and were added to autologous Tconv cell cultures at different ratios (Tconv cells/Treg ratio $=1: 1,2: 1$, and 4:1). CFSE dilution was evaluated at day 5 .

High-dimensional flow cytometry data analysis. Flow Cytometry Standard (FCS) 3.0 files were imported into FlowJo software (version 9) and analyzed by standard gating to remove aggregates and dead cells and identify $\mathrm{CD}^{+} \mathrm{CD} 4^{+}$or $\mathrm{CD} 8^{+} \mathrm{T}$ cells. Three thousand $\mathrm{CD} 4^{+}$ and $\mathrm{CD}^{+} \mathrm{T}$ cells per sample were subsequently imported in FlowJo (version 10), biexponentially transformed, and exported for further analysis in Python (version 3.7.3) by a custom-made pipeline of PhenoGraph (available here http://github.com/luglilab/Cytophenograph, where we modified the linux-community and the core.py script of PhenoGraph package in order to fix the seed to "123456"). Blood, adjacent lung tissue, and tumor samples were labeled with a unique computational barcode for further identification and converted in comma separated (CSV) files and concatenated in a single matrix by using the merge function of pandas package. The $\mathrm{K}$ value, indicating the number of nearest neighbors identified in the first iteration of the algorithm, was set at 40 and 60 for $\mathrm{CD}^{+}$and $\mathrm{CD} 8^{+} \mathrm{T}$ cells clustering, respectively. Clusters representing less than $1 \%$ of total $\mathrm{CD} 4^{+}$or $\mathrm{CD} 8^{+}$ $\mathrm{T}$ cells were removed in subsequent analysis. The data were then reorganized and saved as new CSV files, one for each cluster, that were further analyzed in FlowJo to determine the frequency of positive cells for each marker and the corresponding median fluorescent intensity (MFI). These values were multiplied to derive the iMFI (rescaled to values from 0 to 100; Figure $4 \mathrm{~B}$ and refs. 24,39 ) and then visualized in a heatmap. Subsequent metaclustering of iMFI values was performed using the gplots R package. Hierarchical metaclustering of all samples, based on the frequency of PhenoGraph clusters (Figure 4B), was performed in R according to the Ward minimum variance method. UMAP was obtained by UMAP Python package and visualized in FlowJo 10.

Melanoma data set. Normalized scRNA-seq counts were retrieved from the Gene Expression Omnibus (GEO GSE72056). Analysis was restricted to the cells labeled as "T cells," as previously defined by Tirosh et al. (27). T cells were divided into $\mathrm{CD}^{+}$and $\mathrm{CD} 8^{+}$, based on the normalized expression levels (E) of CD4 (E > 4) and CD8 (average of $C D 8 A$ and $C D 8 B, E>4$ ). Among all samples composing the data set, 466 were identified as $\mathrm{CD} 4^{+}$. To asses which genes have the expression profile most correlated with that of IRF4, a Pearson correlation coefficient, indicated as score in Supplemental Figure 1B, and a corresponding $P$ value were computed between each gene expression profile in the matrix (consisting of 23,686 genes) and IRF4. In order to obtain the CD8 signature used in Supplemental Figure 5B, DEGs in the pairwise comparison between $\mathrm{CD}^{+}$and $\mathrm{CD} 4^{+} \mathrm{T}$ cell subsets were determined by the "FindAllMarkers" function coded in the Seurat $\mathrm{R}$ package (version 2.3.4) with default parameters (54). In this way, we obtained 225 specific genes for the $\mathrm{CD} 8^{+}$subpopulation respect to the $\mathrm{CD} 4^{+} \mathrm{T}$ cells.

Lung and liver data sets. We took advantage of the web server for exploration of NSCLC (http://lung.cancer-pku.cn) and hepatocellular carcinoma (http://hcc.cancer-pku.cn) single T cell RNA-seq data to assess the expression levels of markers of interest within the $\mathrm{CD} 4^{+}$intratumoral subpopulations. Lung and liver tumor-infiltrating $\mathrm{CD}^{+}$signatures (Figure 5D and Supplemental Figure 5A) were obtained by combining all the DEGs from the CD $8^{+}$tissue-specific clusters and by further excluding the circulating clusters labeled as CD8-C1-LEF1 $(26,27)$.

Mice. Irf $4^{-/-}$mice (55) were originally provided by Tak Mak (Princess Margaret Cancer Centre, University Health Network, Toronto, Canada). Irf $4^{-/-}$mice were crossed to Foxp $3^{\text {RFP }}$ mice. Foxp $3^{\text {EGFP-Cre-ERT2 }}$ mice (JAX 016961) (56) were crossed to Irf4-floxed mice (JAX 009380) (57) to allow for specific deletion of Irf4 in Foxp3 ${ }^{+}$cells following tamoxifen treatment.

Mouse procedures. Mice were irradiated $(2 \times 5.5 \mathrm{~Gy})$ and reconstituted by i.v. injection of $200 \mu \mathrm{L}$ bone marrow isolated from femurs and tibias of donor mice. Following irradiation mice were treated with neomycin in drinking water for 4 weeks and allowed to recover for at least 8 weeks. MC38 tumor cells (provided by Stephen Nutt, Walter and Eliza Hall Institute of Medical Research, Melbourne, Victoria, Australia) were grown in DMEM with $10 \%$ FCS and 1\% PenStrep (Gibco) in $37^{\circ} \mathrm{C}, 5 \% \mathrm{CO}_{2}$ incubator and passaged every 2 to 3 days. For tumor inoculation, $1 \times 10^{6}$ cells were resuspended in $100 \mu \mathrm{l}$ PBS and injected subcutaneously into the right flank of the mice. Tumor growth 
was assessed with LCD Digital Vernier Calipers (Protech) every 2 to 3 days. Tamoxifen ( $2 \mathrm{mg} /$ mouse) was administered in $200 \mu \mathrm{L}$ sunflower oil i.p. for 5 consecutive days. Upon the experimental endpoint, mice were euthanized with $\mathrm{CO}_{2}$ and cervical dislocation, and tumors were excised with forceps and scissors. Tumors were mechanically dissociated and digested in $1 \mathrm{mg} / \mathrm{mL}$ Collagenase IV (Gibco) in RPMI medium for 45 minutes at $37^{\circ} \mathrm{C}$, with constant agitation.

Treg isolation from mice. Single-cell suspensions from spleens were enriched for $\mathrm{CD}^{+} \mathrm{T}$ cells by depleting $\mathrm{CD} 8^{+} \mathrm{T}$ cells and $\mathrm{B}$ cells using anti-CD8 and $\mathrm{B} 220$ antibodies. The $\mathrm{CD} 4^{+} \mathrm{T}$ cell-enriched cell suspension was stained with CD4 (RM 4-5) and TCR $\beta$ (H57-597) antibodies. $\mathrm{CD} 4^{+} \mathrm{TCR} \beta^{+}$Foxp $3 \mathrm{RFP}^{+}$cells were sorted from Foxp $3^{\text {RFP }}(\mathrm{WT})$ and $\mathrm{Irf}^{4^{-/}}$ Foxp $3^{\text {RFP }}$ mice.

Mouse RNA isolation and sequencing. RNA purification was performed following the manufacturer's protocol using the RNAeasy Plus Mini Kit (Qiagen). RNA from WT and Irf4 $4^{--}$Tregs was sequenced using Illumina platform (75-bp paired-end reads).

RNA-seq and bioinformatic analysis. RNA isolation of the FACS-purified CCR8 ${ }^{+} \mathrm{ICOS}^{+}$and CCR8- ${ }^{-} \mathrm{ICOS}^{-}$Tregs was performed following the manufacturer's protocol using the Quick-RNA Microprep kit (Zymo Research). RNA quality control was performed with the Agilent 2200 Tape Station system, and only RNAs having a RIN $>8$ were used for library preparation. Libraries for mRNA sequencing were prepared starting from $1.5 \mathrm{ng}$ total RNA for each sample by using the SMART-Seq v4 Ultra Low Input RNA Kit (Clontech-Takara). All samples were sequenced on an Illumina NextSeq 500 at an average of 32.9 million 75-bp single-end reads. After quality control, raw reads were aligned to the human genome (GRCh38.p12) using the STAR aligner with default parameters (version 2.7.0) (58). Gene-based read counts were then obtained using HTSeq count (59) module (version 0.11) and GENCODE v29.gtf annotation (60). The read counts were imported into R statistical software, and differential gene expression analysis was performed using the edgeR package (version 3.22) (61). For pair-wise comparisons, raw read counts were normalized using the TMM method (trimmed mean of log-ratio values) (62), and genes that failed to achieve a counts per million (CPM) mapped reads value greater than 1 in at least 2 libraries were not considered. $P$ values were adjusted using the Benjamini-Hochberg method. Genes were considered differentially expressed when FDR $<0.05$ and when they had an expression change of more than $1 \log _{2}$ fold change. The heatmap representing the $\log _{2}$ of CPM was obtained using pheatmap R package (version 1.0.12) with the distance method "correlation" for both rows and columns.

Gene signature identification (RNA-seq). GSEA was performed using GSEA (version 3.0) software (Broad Institute of MIT) and gene list ranked based on $\log _{2}$ fold changes. The gene set enrichment analysis was conducted in preranked mode with scoring scheme "classic" and 1,000 permutations. The maximum gene set size was fixed at 5,000 genes, and the minimum size was fixed at 10 genes. The gene signature was retrieved from the $\mathrm{H}$ collection (h.all.v6.2.symbols.gmt) of the Molecular Signatures Database (MSigDB v6.2). The GSEA in Supplemental Figure 3B was performed with custom gene sets relevant to immunological signatures (https://github.com/luglilab/ProjectScripts_Treg_Irf4/blob/master/Figure3S/Mouse_ImmunePath_ February_01_2019_symbol.gmt). The dot plot was generated with a custom Rscript (https://github.com/luglilab/ProjectScripts_Treg_ Irf4/blob/master/Figure3S/ImmunoSignatureDotPlot.r).
Motif enrichment analysis. The PScan software tool (version 1.5) was used to perform the in silico computational analysis of overrepresented TF binding sites within the 5 '-promoter regions of DEGs (63). PScan was run on $[-950,+50]$ bp upstream regions onto the Homo Sapiens JASPAR 2018_NR database (64). Results were summarized with a scatter plot, where $P$ values were plotted against $Z$ score on vertical axis by using the Python Matplotlib package (version 3.0.3).

Bioinformatic analysis of microarray data. Microarray probe fluorescence signals downloaded from the Gene Expression Omnibus (GEO GSE89656 and GSE61077, samples GSM1496276, GSM1496277, GSM1496274, GSM1496275) were converted to expression values using robust multiarray average procedure RMA (65) of the Bioconductor Affy package. Fluorescence intensities were background adjusted and normalized using quantile normalization, and expression values were calculated using median polish summarization and custom chip definition files for a total of 18,075 custom probe sets for Mouse Genome 430 2.0 Array based on Entrez genes (Mouse4302_Mm_ENTREZG version 21.0.0) and 12,426 custom probe sets for Mouse Genome 430A 2.0 Array based on Entrez genes (Mouse430A2_Mm_ENTREZG version 21.0.0). To identify genes that are differentially expressed, we compared the expression profiles of

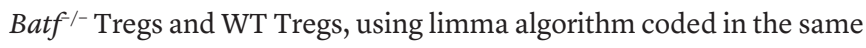
R package (66). All data analyses were performed in $\mathrm{R}$ (version 3.4.4) using Bioconductor libraries and R statistical packages.

Bioinformatic analysis of ChIP-seq data. Raw data were downloaded from the Gene Expression Omnibus (GEO GSE98264). Reads were aligned to mouse genome GRCm38.p6/mm10 using Bowtie2 (version 2.1.0) (67) in local alignment mode. After alignment to the reference genome, mitochondrial and ambiguously mapped reads were discarded with Samtools (68) and further used for sorting and indexing mapping files. Bigwig files for IGV (69) genome coverage visualization were generated with the multiBamSummary module from the deepTools suite (version 3.2.0) (70). To call peaks, we used MACS2 (version 2.1.2) (71) with these parameters: callpeak gsize mm nomodel extsize 147 and $Q$ value $1 \times 10^{-3}$. Peaks within $30 \mathrm{~kb}$ upstream and $10 \mathrm{~kb}$ downstream of the TSS or within intragenic regions were annotated with the closest TSS using ChIPSeeker (version 1.18.0) (72) and GENCODE M20.gtf gene annotation.

Survival analysis. Transcriptomic and clinical data of human lung adenocarcinoma, hepatocellular carcinoma, and melanoma from TCGA database (provisional cohorts) were obtained from the cBioPortal platform. Signatures of CCR $8^{+} \mathrm{ICOS}^{+}$Tregs (Figure 2B) and CD ${ }^{+} \mathrm{T}$ cells (see "Gene signature identification") were used to calculate patient-specific enrichment scores from specimens of lung adenocarcinoma (LUAD), hepatocellular carcinoma (LIHC) and melanoma (SKCM) data sets ("GSVA" R package) (73). Survival curves were calculated between groups of patients subdivided according to the percentile rank (set at 0.8) of the resulting scores. The R packages "survival" (http://cran.rproject.org/web/packages/survival/index.html) and "survminer" (http:// cran.r-project.org/web/packages/survminer/index.html) were used to assess statistics and obtain survival curves.

Data availability. The gene expression data generated in this study are available in the GEO database (GSE128822).

Statistics. Statistical analyses were performed using GraphPad Prism (version 7), unless specified otherwise. Data were first tested for normal distribution with D'Agostino-Pearson, Shapiro-Wilk, or Kolmogorov-Smirnov normality tests and then analyzed with Student's $t$ 
test (paired, 2 tailed), Wilcoxon rank test (paired nonparametric data), or Mann-Whitney (unpaired nonparametric data) when comparing 2 groups or Friedman test or 2-way ANOVA with Bonferroni's post hoc test for multiple comparisons when comparing 3 groups (specific tests are specified in the figure legends).

Correlation of gene expression from scRNA-seq data was determined by calculating the Pearson correlation coefficient. $P$ values of less than or equal to 0.05 were considered significant. PCA was performed by the prcomp function of the R stats package. Hypergeometric tests between Irf4 or Batf DEGs or scRNA-seq signature (25) and the murine tumor-infiltrating Treg signature (Figure $3 \mathrm{~B}$ ) were run with GeneOverlap R package.

Study approval. All human experiments were approved by the Humanitas Clinical and Research Center Internal Review Board (approval 1501). All patients provided written informed consent. All mice were maintained and used in accordance with the guidelines of the University of Melbourne Animal Ethics Committee.

\section{Author contributions}

GA, J. Brummelman, SP, EMCM, EPT, CP, AV, and J. Blume performed experiments. FSC provided expertise with cell sorting. VZ, CP, and AS provided technical support. MA, PN, and GV performed surgery. AL, E. Lopci, and GV provided clinical information and helped with clinical interpretation of the data. RR, MK, and MP provided expertise in data analysis. GA, J. Brummelman, SP, AK, and E. Lugli conceived the study. E. Lugli supervised the study. GA and E. Lugli wrote the paper. All authors edited the paper. GA, J. Brummelman, and SP share first authorship. GA and J. Brummelman provided equal contribution in conceiving the overall study and in performing the vast majority of the experiments, while SP conceived and performed all the bioinformatic analyses. Given the importance of bioinformatics in this manuscript, these authors deserve equal contribution. The order of the first authors reflects the leadership exerted in the study.

\section{Acknowledgments}

We wish to thank Elisa Dieci (Humanitas Clinical and Research Center) for helping with patients' information, Gianluca Rotta and Jens Fleischer (BD Biosciences) for helping with FACSymphony A5 instrument setup, as well as Silvia Piconese (University of Rome La Sapienza), Gioacchino Natoli (European Institute of Oncology), and the members of the Laboratory of Translational Immunology for critical discussion. This work has been supported by grants from the Associazione Italiana per la Ricerca sul Cancro (investigator grant 20676 to E. Lugli and My First AIRC Grant 189323 to E. Lopci), Bristol-Myers Squibb (preclinical grant CA209-9YM to E. Lugli), and the Humanitas Clinical and Research Center. J. Brummelman is a recipient of the "Fondo di beneficenza Intesa San Paolo" fellowship from AIRC. EMCM and PN are recipients of the 2017 Fondazione Umberto Veronesi postdoctoral fellowship. Purchase of the BD FACSymphony A5 has been in part defrayed by a grant from Italian Ministry of Health (agreement 82/2015). AK is supported by grants and a fellowship from the National Health and Medical Research Council of Australia. J. Blume was supported by a fellowship from the Deutsche Forschungsgemeinschaft.

Address correspondence to: Enrico Lugli, Via Manzoni 56, 20089, Rozzano, Italy. Phone: 39.02.8224.5143; Email: enrico.lugli@ humanitasresearch.it.
1. Speiser DE, Ho PC, Verdeil G. Regulatory circuits of $\mathrm{T}$ cell function in cancer. Nat Rev Immunol. 2016;16(10):599-611.

2. Tanaka A, Sakaguchi S. Regulatory T cells in cancer immunotherapy. Cell Res. 2017;27(1):109-118.

3. Wing K, et al. CTLA-4 control over

Foxp3+ regulatory $\mathrm{T}$ cell function. Science. 2008;322(5899):271-275.

4. Larmonier N, et al. Tumor-derived CD4(+) $\mathrm{CD} 25(+)$ regulatory $\mathrm{T}$ cell suppression of dendritic cell function involves TGF-beta and IL-10. Cancer Immunol Immunother. 2007;56(1):48-59.

5. Chen ML, et al. Regulatory T cells suppress tumor-specific CD8 T cell cytotoxicity through TGF-beta signals in vivo. Proc Natl Acad Sci USA. 2005;102(2):419-424.

6. Collison LW, et al. The inhibitory cytokine IL-35 contributes to regulatory T-cell function. Nature. 2007;450(7169):566-569.

7. Deaglio S, et al. Adenosine generation catalyzed by CD39 and CD73 expressed on regulatory $\mathrm{T}$ cells mediates immune suppression. J Exp Med. 2007;204(6):1257-1265.

8. Grossman WJ, Verbsky JW, Barchet W, Colonna M, Atkinson JP, Ley TJ. Human T regulatory cells can use the perforin pathway to cause autologous target cell death. Immunity. 2004;21(4):589-601.

9. Vignali DA, Collison LW, Workman CJ. How regulatory T cells work. Nat Rev Immunol. 2008;8(7):523-532.
10. Roychoudhuri R, Eil RL, Restifo NP. The interplay of effector and regulatory $\mathrm{T}$ cells in cancer. Curr Opin Immunol. 2015;33:101-111.

11. Wei WZ, et al. Concurrent induction of antitumor immunity and autoimmune thyroiditis in CD4+ CD25+ regulatory T cell-depleted mice. Cancer Res. 2005;65(18):8471-8478.

12. Dannull J, et al. Enhancement of vaccine-mediated antitumor immunity in cancer patients after depletion of regulatory T cells. J Clin Invest. 2005;115(12):3623-3633.

13. Colombo MP, Piconese S. Regulatory-T-cell inhibition versus depletion: the right choice in cancer immunotherapy. Nat Rev Cancer. 2007;7(11):880-887.

14. Luo CT, Liao W, Dadi S, Toure A, Li MO. Graded Foxo1 activity in Treg cells differentiates tumour immunity from spontaneous autoimmunity. Nature. 2016;529(7587):532-536.

15. De Simone M, et al. Transcriptional landscape of human tissue lymphocytes unveils uniqueness of tumor-infiltrating T regulatory cells. Immunity. 2016;45(5):1135-1147.

16. Plitas G, et al. Regulatory T cells exhibit distinct features in human breast cancer. Immunity. 2016;45(5):1122-1134.

17. Cretney E, Kallies A, Nutt SL. Differentiation and function of Foxp3(+) effector regulatory T cells. Trends Immunol. 2013;34(2):74-80.

18. Cipolletta D, et al. PPAR- $\gamma$ is a major driver of the accumulation and phenotype of adipose tissue Treg cells. Nature. 2012;486(7404):549-553.

19. Zheng Y, et al. Regulatory T-cell suppressor program co-opts transcription factor IRF 4 to control $\mathrm{T}(\mathrm{H}) 2$ responses. Nature. 2009;458(7236):351-356.

20. Cretney E, et al. The transcription factors Blimp-1 and IRF4 jointly control the differentiation and function of effector regulatory T cells. Nat Immunol. 2011;12(4):304-311.

21. Li P, et al. BATF-JUN is critical for IRF4-mediated transcription in T cells. Nature. 2012;490(7421):543-546.

22. Tussiwand $\mathrm{R}$, et al. Compensatory dendritic cell development mediated by BATF-IRF interactions. Nature. 2012;490(7421):502-507.

23. Akimova T, et al. Human lung tumor FOXP3+ Tregs upregulate four "Treg-locking" transcription factors. JCI Insight. 2017;2(16):94075.

24. Brummelman J, et al. High-dimensional single cell analysis identifies stem-like cytotoxic $\mathrm{CD}^{+}$ T cells infiltrating human tumors. J Exp Med. 2018;215(10):2520-2535

25. Guo X, et al. Global characterization of T cells in non-small-cell lung cancer by single-cell sequencing. Nat Med. 2018;24(7):978-985.

26. Zheng C, et al. Landscape of infiltrating T cells in liver cancer revealed by single-cell sequencing. Cell. 2017;169(7):1342-1356.e16.

27. Tirosh I, et al. Dissecting the multicellular ecosystem of metastatic melanoma by single-cell 
RNA-seq. Science. 2016;352(6282):189-196.

28. Kim HJ, et al. Stable inhibitory activity of regulatory $\mathrm{T}$ cells requires the transcription factor Helios. Science. 2015;350(6258):334-339.

29. Wei X, et al. Reciprocal expression of IL-35 and IL-10 defines two distinct effector Treg subsets that are required for maintenance of immune tolerance. Cell Rep. 2017;21(7):1853-1869.

30. Roychoudhuri R, et al. BACH2 represses effector programs to stabilize $\mathrm{T}(\mathrm{reg})$-mediated immune homeostasis. Nature. 2013;498(7455):506-510.

31. Chapman NM, et al. mTOR coordinates transcriptional programs and mitochondrial metabolism of activated $\mathrm{T}_{\text {reg }}$ subsets to protect tissue homeostasis. Nat Commun. 2018;9(1):2095.

32. Pilipow K, et al. Antioxidant metabolism regulates $\mathrm{CD} 8+\mathrm{T}$ memory stem cell formation and antitumor immunity. JCI Insight. 2018;3(18):122299.

33. Hirotsu Y, Hataya N, Katsuoka F, Yamamoto M. NF-E2-related factor 1 (Nrf1) serves as a novel regulator of hepatic lipid metabolism through regulation of the Lipin1 and PGC-1 $\beta$ genes. Mol Cell Biol. 2012;32(14):2760-2770.

34. Wang R, et al. The transcription factor Myc controls metabolic reprogramming upon $\mathrm{T}$ lymphocyte activation. Immunity. 2011;35(6):871-882.

35. Vasanthakumar A, et al. The TNF receptor superfamily-NF- $\kappa \mathrm{B}$ axis is critical to maintain effector regulatory T cells in lymphoid and non-lymphoid tissues. Cell Rep. 2017;20(12):2906-2920.

36. Magnuson AM, et al. Identification and validation of a tumor-infiltrating Treg transcriptional signature conserved across species and tumor types. Proc Natl Acad Sci USA. 2018;115(45):E10672-E10681.

37. Hayatsu N, et al. Analyses of a mutant Foxp3 allele reveal BATF as a critical transcription factor in the differentiation and accumulation of tissue regulatory T cells. Immunity. 2017;47(2):268-283.e9.

38. Kallikourdis M, Andersen KG, Welch KA, Betz AG. Alloantigen-enhanced accumulation of CCR5+ 'effector' regulatory $\mathrm{T}$ cells in the gravid uterus. Proc Natl Acad Sci USA. 2007;104(2):594-599.

39. Brummelman J, et al. Development, application and computational analysis of high-dimensional fluorescent antibody panels for single-cell flow cytometry. Nat Protoc. 2019;14(7):1946-1969.

40. Levine JH, et al. Data-driven phenotypic dissection of AML reveals progenitor-like cells that correlate with prognosis. Cell. 2015;162(1):184-197.

41. Li J, He Y, Hao J, Ni L, Dong C. High levels of eomes promote exhaustion of anti-tumor $\mathrm{CD}^{+}$ T cells. Front Immunol. 2018;9:2981.
42. Galon J, Angell HK, Bedognetti D, Marincola FM. The continuum of cancer immunosurveillance: prognostic, predictive, and mechanistic signatures. Immunity. 2013;39(1):11-26.

43. Cancer Genome Atlas Research Network. Comprehensive molecular profiling of lung adenocarcinoma. Nature. 2014;511(7511):543-550.

44. Cancer Genome Atlas Research Network. Electronic address: wheeler@bcm.edu, Cancer Genome Atlas Research Network. Comprehensive and Integrative Genomic Characterization of Hepatocellular Carcinoma. Cell. 2017;169(7):1327-1341.e23.

45. Levine AG, Arvey A, Jin W, Rudensky AY. Continuous requirement for the TCR in regulatory $\mathrm{T}$ cell function. Nat Immunol. 2014;15(11):1070-1078.

46. Glasmacher E, et al. A genomic regulatory element that directs assembly and function of immune-specific AP-1-IRF complexes. Science. 2012;338(6109):975-980.

47. Koizumi SI, et al. JunB regulates homeostasis and suppressive functions of effector regulatory $\mathrm{T}$ cells. Nat Commun. 2018;9(1):5344.

48. Schaefer TS, Sanders LK, Nathans D. Cooperative transcriptional activity of Jun and Stat 3 beta, a short form of Stat3. Proc Natl Acad Sci USA. 1995;92(20):9097-9101.

49. Li A, et al. IL-33 signaling alters regulatory $\mathrm{T}$ cell diversity in support of tumor development. Cell Rep. 2019;29(10):2998-3008.e8.

50. Wang L, et al. Connecting blood and intratumoral $\mathrm{T}_{\text {reg }}$ cell activity in predicting future relapse in breast cancer. Nat Immunol. 2019;20(9):1220-1230.

51. Cavalleri T, et al. Combined low densities of FoxP3 ${ }^{+}$ and $\mathrm{CD}^{+}$tumor-infiltrating lymphocytes identify stage II colorectal cancer at high risk of progression. Cancer Immunol Res. 2019;7(5):751-758.

52. Di Pilato M, et al. Targeting the CBM complex causes $\mathrm{T}_{\text {reg }}$ cells to prime tumours for immune checkpoint therapy. Nature. 2019;570(7759):112-116.

53. Lugli E, Zanon V, Mavilio D, Roberto A. FACS analysis of memory T lymphocytes. Methods $\mathrm{Mol}$ Biol. 2017;1514:31-47.

54. Satija R, Farrell JA, Gennert D, Schier AF, Regev A. Spatial reconstruction of single-cell gene expression data. Nat Biotechnol. 2015;33(5):495-502.

55. Mittrücker HW, et al. Requirement for the transcription factor LSIRF/IRF4 for mature B and T lymphocyte function. Science. 1997;275(5299):540-543.

56. Rubtsov YP, et al. Stability of the regulatory $\mathrm{T}$ cell lineage in vivo. Science. 2010;329(5999):1667-1671.

57. Klein U, et al. Transcription factor IRF4 controls plasma cell differentiation and class-switch recom- bination. Nat Immunol. 2006;7(7):773-782.

58. Dobin A, et al. STAR: ultrafast universal RNA-seq aligner. Bioinformatics. 2013;29(1):15-21.

59. Anders S, Pyl PT, Huber W. HTSeq--a Python framework to work with high-throughput sequencing data. Bioinformatics. 2015;31(2):166-169.

60. Frankish A, et al. GENCODE reference annotation for the human and mouse genomes. Nucleic Acids Res. 2019;47(D1):D766-D773.

61. Robinson MD, McCarthy DJ, Smyth GK. edgeR: a Bioconductor package for differential expression analysis of digital gene expression data. Bioinformatics. 2010;26(1):139-140.

62. Robinson MD, Oshlack A. A scaling normalization method for differential expression analysis of RNA-seq data. Genome Biol. 2010;11(3):R25.

63. Zambelli F, Pesole G, Pavesi G. Pscan: finding over-represented transcription factor binding site motifs in sequences from co-regulated or co-expressed genes. Nucleic Acids Res. 2009;37(Web Server issue):W247-W252.

64. Khan A, et al. JASPAR 2018: update of the open-access database of transcription factor binding profiles and its web framework. Nucleic Acids Res. 2018;46(D1):D260-D266.

65. Irizarry RA, et al. Exploration, normalization, and summaries of high density oligonucleotide array probe level data. Biostatistics. 2003;4(2):249-264.

66. Ritchie ME, et al. limma powers differential expression analyses for RNA-sequencing and microarray studies. Nucleic Acids Res. 2015;43(7):e47.

67. Langmead B, Wilks C, Antonescu V, Charles R. Scaling read aligners to hundreds of threads on general-purpose processors. Bioinformatics. 2019;35(3):421-432.

68. Li H, et al. The Sequence Alignment/ Map format and SAMtools. Bioinformatics. 2009;25(16):2078-2079.

69. Robinson JT, et al. Integrative genomics viewer. Nat Biotechnol. 2011;29(1):24-26.

70. Ramírez F, et al. deepTools2: a next generation web server for deep-sequencing data analysis. Nucleic Acids Res. 2016;44(W1):W160-W165.

71. Zhang Y, et al. Model-based analysis of ChIP-Seq (MACS). Genome Biol. 2008;9(9):R137.

72. Yu G, Wang LG, He QY. ChIPseeker: an R/Bioconductor package for ChIP peak annotation, comparison and visualization. Bioinformatics. 2015;31(14):2382-2383.

73. Hänzelmann S, Castelo R, Guinney J. GSVA: gene set variation analysis for microarray and RNAseq data. BMC Bioinformatics. 2013;14:7. 